\title{
Vibration measurements of a wire scanner - experimental setup and models
}

\author{
Juan Herranz ${ }^{a, b, c}$, Ana Barjau ${ }^{b}$, Bernd Dehning ${ }^{a}$ \\ (a) CERN, CH-1211 Geneva 23 - Switzerland, \\ (b) Universitat Politècnica de Catalunya, Diagonal 647, 08028 Barcelona - Spain, \\ (c) Proactive R\&D, Diagonal 429, 08036 Barcelona - Spain. \\ Corresponding author: Juan Herranz, phone: +41 7678 38439, fax: +41 2276 69244, \\ email: juan.herranz.alvarez@cern.ch
}

\begin{abstract}
In the next years the luminosity of the LHC will be significantly increased. This will require a much higher accuracy of beam profile measurement than actually achievable by the current wire scanner. The new performance demands a wire travelling speed up to $20 \mathrm{~m} \cdot \mathrm{s}^{-1}$ and a position measurement accuracy of the order of $1 \mu \mathrm{m}$. The vibrations of the mechanical parts of the system, and particularly the vibrations of the thin carbon wire, have been identified as the major error sources of wire position uncertainty. Therefore the understanding of the wire vibrations has been given high priority for the design and operation of the new device. This article presents a new strategy to measure the wire vibrations based on the piezoresistive effect of the wire itself. An electronic readout system based on a Wheatstone bridge is used to measure the variation of the carbon wire resistance, which is directly proportional to the wire elongation caused by the oscillations.
\end{abstract}

Keywords: wire scanner; wire vibrations; vibration measurements; calibration; FE analysis; piezoresistive; strain gauges

\section{INTRODUCTION}

A wire scanner is an electro-mechanical device which measures the transverse beam profile in a particle accelerator by means of a thin wire moving in an intermittent manner [1]. The intersection of the wire and the beam generates a cascade of secondary particles and scattered primary particles. Those particles are intercepted by a scintillator, coupled with a photomultiplier, which measures the intensity of the light thus produced (Fig. 1). The acquisitions of the wire position and the intensity signal are synchronized with the particle revolution frequency and are combined to construct the transverse beam density profile. Typically for CERN rotating scanners, Proton Synchrotron (PS) and Super Proton Synchrotron (SPS), the wire position is measured with a precision rotary potentiometer. The potentiometer signal and the scintillator photomultiplier signal are digitalized with synchronized ADCs to reconstruct the beam profile. 
The full wire scanner system can be divided into three subsystems: the wire, the actuator (which includes all the mechanical rotating components) and the control system (which includes the controller, the power amplifier and the electrical part of the actuator).

The wire is stretched by a fork directly mounted on a shaft. Wire, fork and shaft are located in a vacuum chamber while the actuator is outside that chamber. The actuator provides a motion pattern consisting of three distinct phases (acceleration, constant speed and deceleration, Fig. 2) fulfilling a set of requirements in order to achieve a suitable wire speed and position at beam crossing [2].

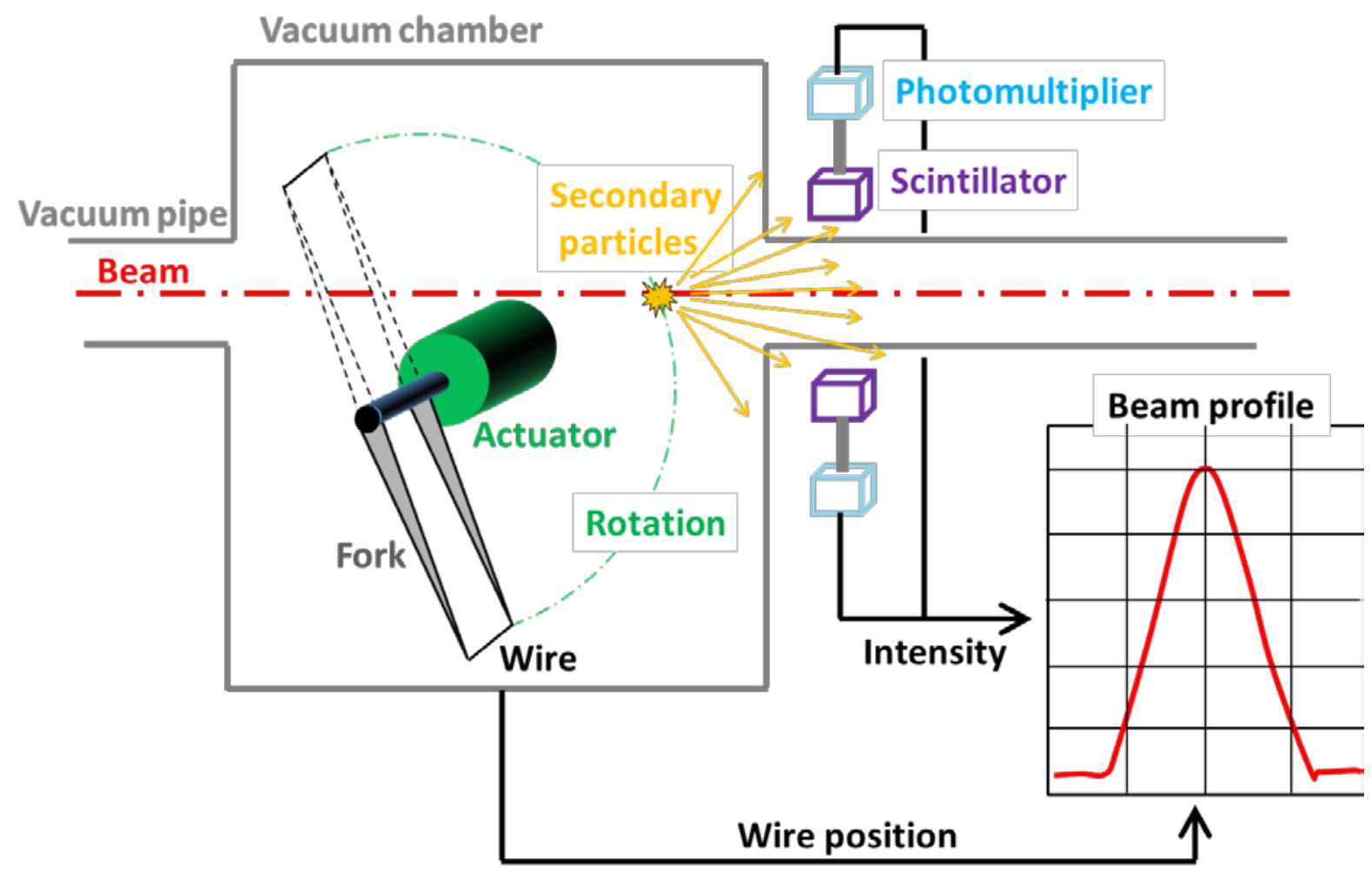

Fig. 1: Schematics of the wire scanner instrument.

Errors on the wire position when intersecting the beam have a direct consequence on the profile and position measurement accuracy. Thus, identifying and minimizing the uncertainties and error sources is a priority issue.

CERN is developing a new wire scanner for all its accelerators and the stronger requirement comes from the LHC because of the beam widths $\sigma$ of about $200 \mu \mathrm{m}$. The aim is to measure beam emittance $\varepsilon$ with a relative accuracy $5 \times 10^{-2}$ i.e. $10 \mu \mathrm{m}$. As $\sigma=\sqrt{ } \beta \varepsilon ; d \sigma / \sigma=d \varepsilon / 2 \varepsilon$, therefore error in the beam width measurement is $1 / 2$ of the wanted error in emittance measurement, i.e, $5 \mu \mathrm{m}$. 
As this error includes all the error contributions of the system, the aim is to make the uncertainty of the error contribution from the wire vibrations amplitude neglectable to the full error.
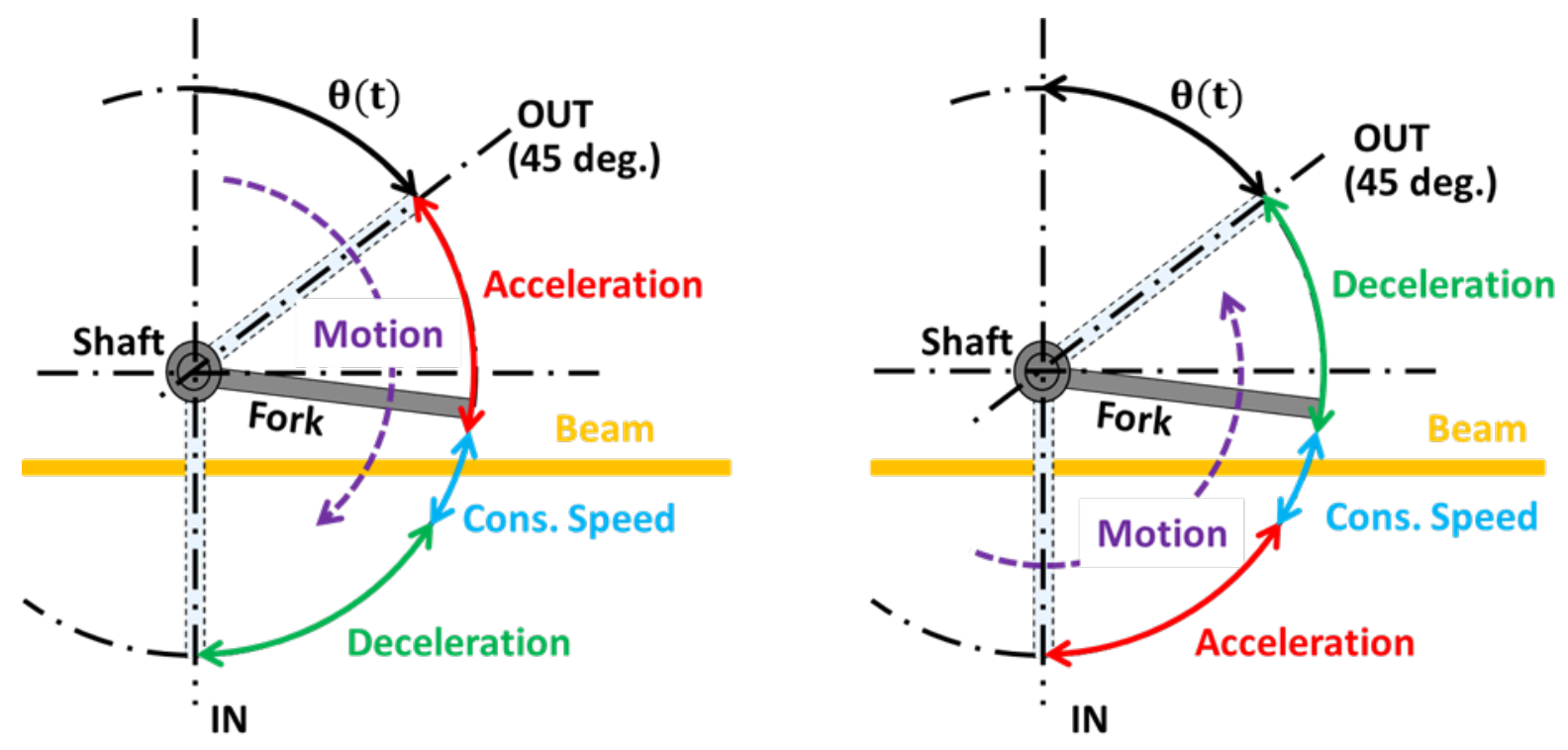

Fig. 2: Schematics of the PS scanner scan cycle from OUT to IN (left) and from IN to OUT (right) positions.

The accuracies achieved in the existing devices are limited by the motorization, the angular position measurement system located outside of the vacuum vessels, and the use of bellows to transfer the movement into the vacuum tank. Furthermore, lever arm based mechanisms used to increase the speed of the wire introduce additional uncertainties [2].

Last but not least, the strong peak acceleration in the motion pattern induces deflections and vibrations on the measurement chain (shaft, fork and wire), which result in discrepancies between the true position of the wire midpoint $(P)$ and the position measured by the angular sensor (point $R$ ) (Fig. 3).

Precision issues are a general problem in measurement devices. For the particular case of wire scanners, they have been addressed by different researchers. Roncarolo [3] performed a thorough study on the accuracy of PS and SPS wire scanners. According to that study, the range of uncertainty may reach $0.2 \mathrm{~mm}$ for the existing scanner in PS and SPS, which means around $10 \%$ relative beam size measurement, because of the lower energy in the LHC injector accelerators and the different $\beta$ functions. This value is in the same order as that presented by Koopman et al. [4] for the PS and SPS scanners. Blokland et al. [5] presented a measured repeatability average of $1 \%$ in the fast wire scanner operating in Tevatron. They also mention some error inflicted by vibration associated with the use of a drive belt and a soft coupler between the encoder and the fork. Bosser 
et al. [6] developed an experimental setup used in the PS and SPS scanner at CERN which showed the wire vibrations during the scan process.

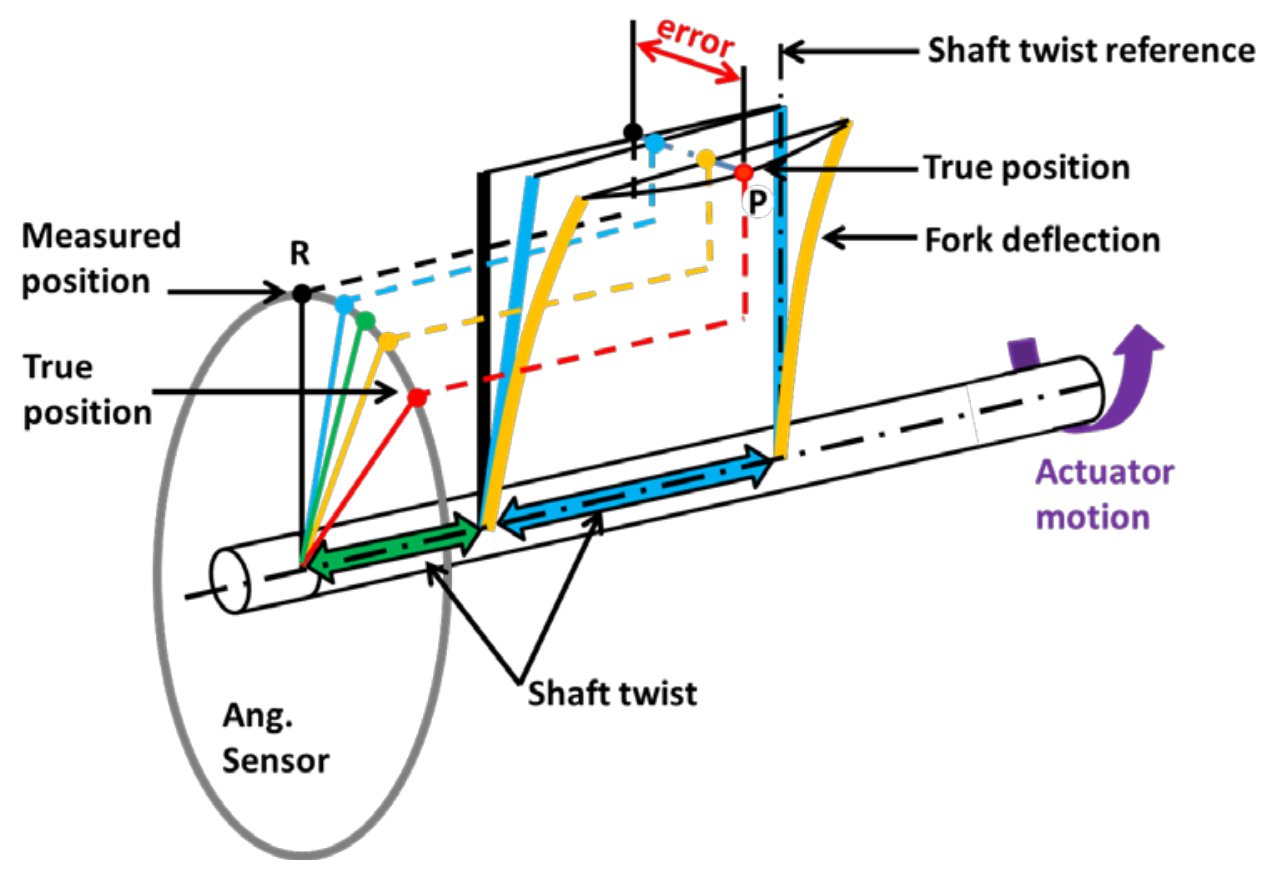

Fig. 3: Schematics of the wire deflection in the measurement chain.

A new design of wire scanner improving the performance of the existing device and overcoming some of those drawbacks has been developed [7]. However, residual vibrations have not been totally eliminated.

The number of accelerators and therefore research groups in beam instrumentation is quite low compared to the number of instrumentation groups in other fields. Consequently existing technologies for vibration measurement are usually not directly linked to the field of beam instrumentation.

Two studies have been found in the literature related specifically to the measurement vibrations in a wire scanner. Bosser et al. [6] proposed a technique based on the voltage induced in a conductor when travelling in a magnetic field. Though implemented to verify the speed of the SPS wire scanner prototype, it revealed the existence of fork oscillations. Therefore, that technique could probably be sensitive to wire oscillations. However that technique requires a very uniform and constant magnetic field that has to be crossed by the wire during its motion. Therefore its implementation in vacuum calls for precise positioning systems. More recently, lida et al. [8] have proposed a method which measures the vibrations on the wire mount frame. The method is based 
on the use of a laser displacement meter located outside the vacuum tank and a reflective flag fixed on the wire holder. This allows measuring the vibration under vacuum (by means of a vacuum view port installed in the vacuum chamber). However, the vibration of the wire itself cannot be measured with this method.

Devices relating different properties of thin wires to some aspects of their vibratory behaviour characteristics exist. Even if the main goal of such devices is not the measurement of the wire vibrations (as they are somehow the tool to measure other parameters), the technology and knowledge to perform measurements in vibrating wires is included in these devices.

Among those devices, the most well-known is probably the Vibrating Wire Stain Gauge (VWSG). This technology uses the vibrational behaviour of a wire to perform strain measurements in structural elements. Nowadays it is a well consolidated technology, and commercial products based on it can be found in the market [9], [10]. This device consists on a stretched wire and a sensor. The sensor contains a permanent magnet and a plucking coil assembly. When the wire is plugged by the sensor, it vibrates at its natural frequency, which is proportional to the tension on the wire. Any change in the wire strain affects directly its tension, resulting in a change in its natural frequency of vibration. Thus the VWSG can provide an indirect stress measurement of a structural element through the multiplication of the measured strain and the modulus of elasticity of element material. The temperature in the VWSG device has a substantial influence on vibration frequency and this effect has to be taken into account [11].

A second interesting device is the Vibrating Wire Scanner (VWS). It is an instrument used to monitor the profile of a beam of particles. Its operating principle is based on the natural frequency change undergone by a stretched wire due to an increase of its temperature. That temperature shift results in a variation in the wire stretching, which in turn produces a change of its natural frequency. When the wire moves across a beam of particles, its temperature will change according to the density of particles. These temperature differences will be read as a change of its natural frequency. Arutunian et al. [12] prove that it is possible to reconstruct the beam profile from that natural frequency change. They also point out that the VWS is very sensitive to the temperature of the base to which the wire is fastened, and that a thermostabilisation of the base is compulsory in order to solve this problem.

This article proposes a new procedure to measure the wire vibrations based on the piezoresistive effect of the wire itself. The main idea is to measure the variation of the carbon wire resistance by means of an electronic readout system based on a Wheatstone bridge. That resistance is directly proportional to the change of wire length due to its vibration. 
The article is organized as follows:

- $\quad$ Section 2 describes the measurement system.

- Section 3 describes the calibration procedures (transversal and longitudinal strain gauges calibration, wire elongation calibration).

- $\quad$ Section 4 is devoted to the measurements of fork and wire vibrations.

- $\quad$ Section 5 presents some qualitative comments on the dynamics of the wire.

- Section 6 contains the conclusions and further developments.

Though the wire dynamics is not the main purpose of this work, we have included a short section of discussion in order to better understand the measurements performed on that element.

\section{MEASUREMENT SYSTEM}

The vibration measurement system has been developed for an existing fast wire scanner used in the PS. The experimental setup consists basically of seven elements (Fig. 4):

- a PS wire scanner actuator,

- a PS wire scanner fork equipped with semiconductor strain gauges,

- an electronic readout system based on a Wheatstone bridge to measure the resistance variation of the strain gauges,

- $\quad$ an electronic readout system based on a Wheatstone bridge to measure the wire resistance variations,

- an oscilloscope,

- a vacuum tank,

- a vacuum pump.

During the scan cycle, the following variables are measured:

- actuator angular position,

- tension on the strain gauges bonded to the fork,

- $\quad$ changes of electrical tension in the carbon wire.

The actuator angular position is measured by means of a precision rotary potentiometer and a 16 bits ADC. This allows an angular resolution of $95 \mu \mathrm{rad}$. 


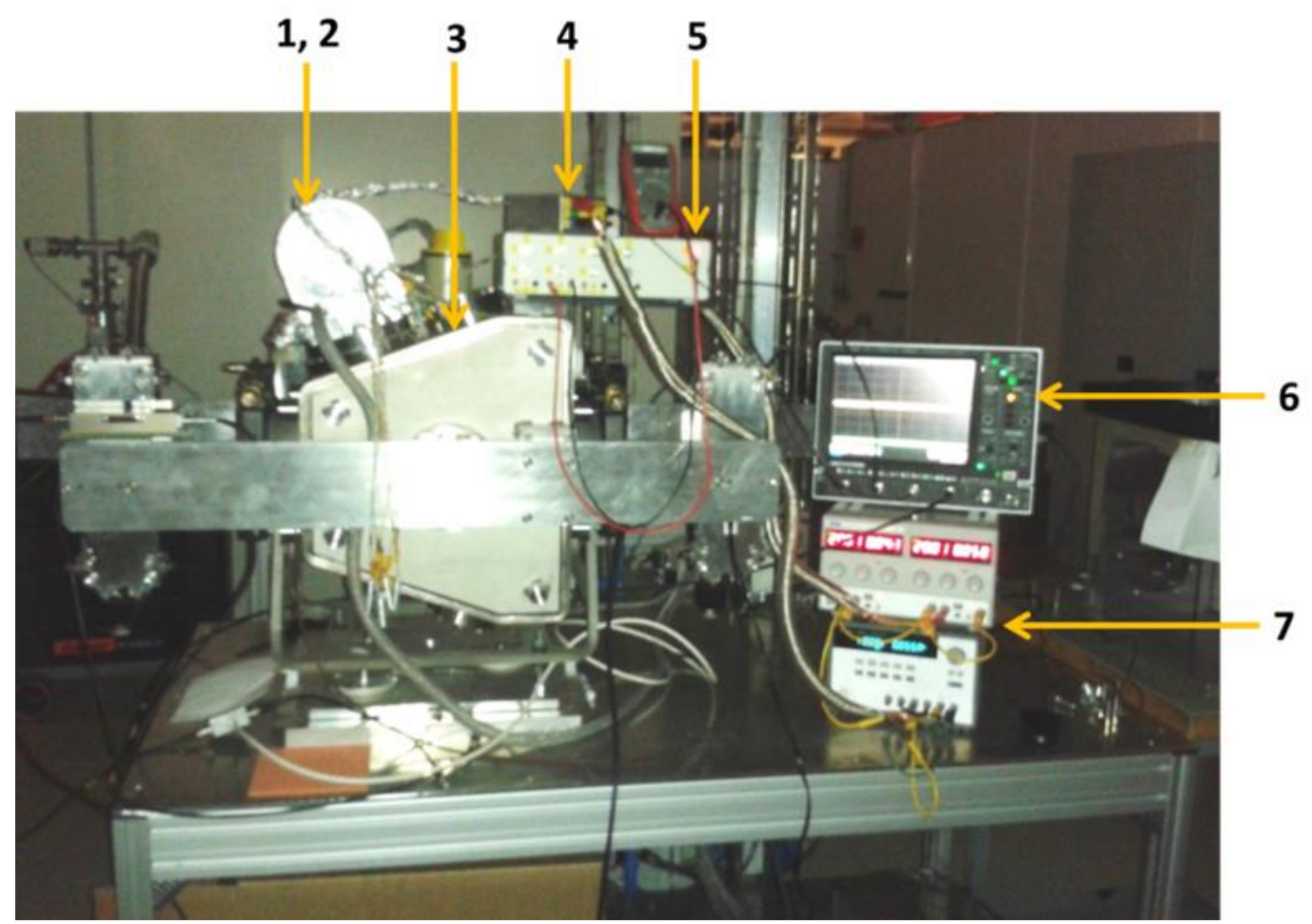

Fig. 4: Experimental setup: actuator (1), fork (2), vacuum tank (3), Wheatstone bridge and amplifier for wire resistance variations (4), Wheatstone bridge and amplifier for strain gauges (5), oscilloscope (6), power supplies (7).

Though the experimental setup would allow monitoring at the same time 8 strain gauges plus the wire resistance variations, the limited number of channels of the oscilloscope (it is a standard one with just 4 channels) allows only the simultaneous recording of 2 strain gauges, the wire resistance variation and the motor angular sensor. Consequently the measurements have to be performed several times in order to cover all the strain gauges.

Although initially standard strain gauges (resistive) were used, their resolution was not enough to show the deflections of the fork arms. They were then replaced by $P$ type silicon (111) semiconductor strain gauges.

Backed semiconductor P type silicon (111) strain gauges have been bonded to some strategic parts of the fork arms using $3 \mathrm{M}^{\mathrm{TM}}$ Scotch-Weld ${ }^{\mathrm{TM}}$ General Purpose Instant Adhesive EC2500 (Fig. 5). The dimensions and performances of the gauge bonded to the fork are shown in Table. 1. 


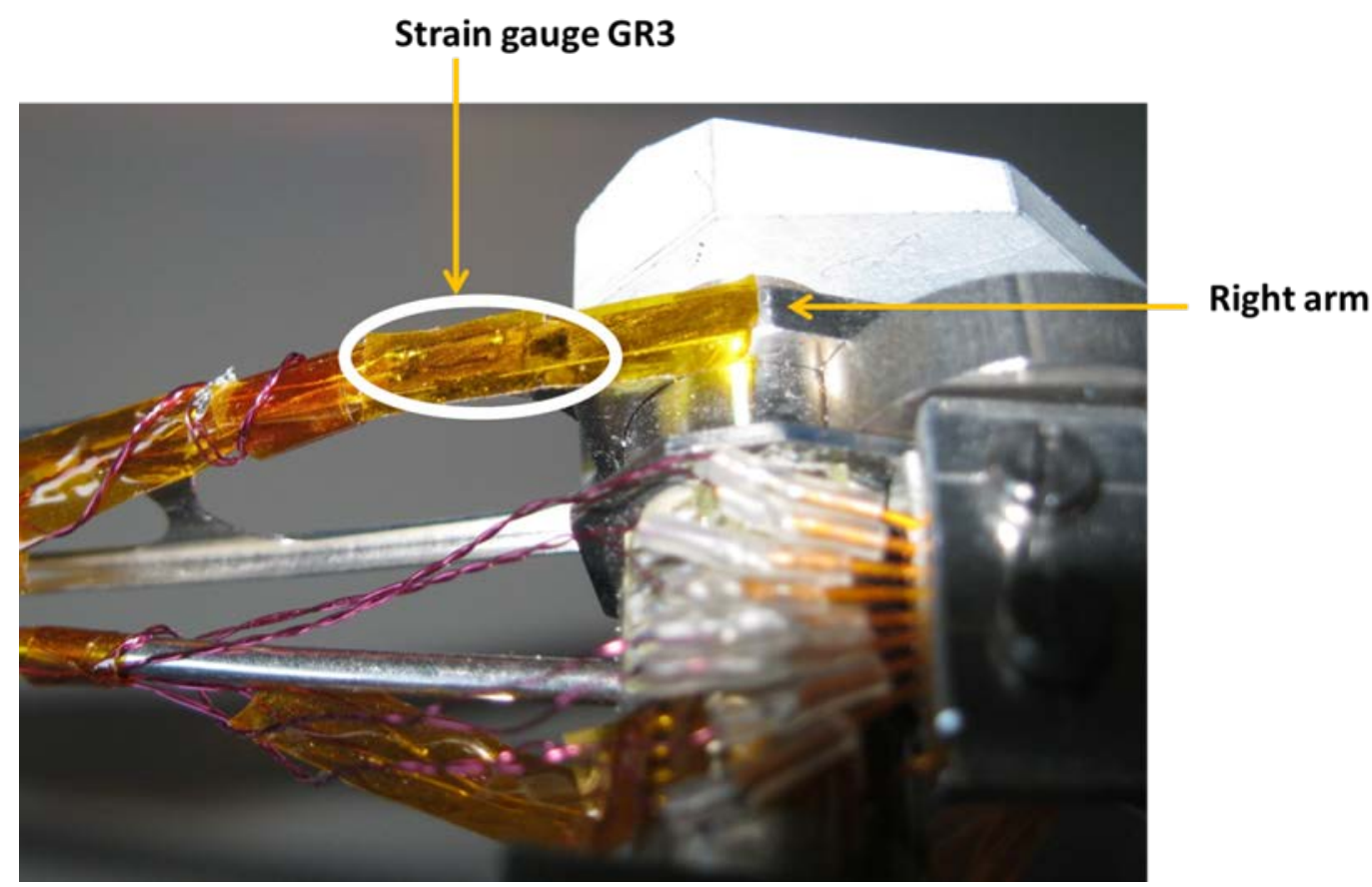

Fig. 5: Semiconductor strain gauge detail bonded in the PS scanner arm.

Table 1: Dimension and performances of semiconductor strain gauges.

\begin{tabular}{|l|l|}
\hline Resistance $[\mathrm{Ohm}]$ & 350 \\
\hline Dimension naked, length $\mathrm{x}$ width $\mathrm{x}$ thickness $[\mathrm{mm}]$ & $3.8 \times 0.2 \times 0.04$ \\
\hline Dimension baking, length $\mathrm{x}$ width $\mathrm{x}$ thickness $[\mathrm{mm}]$ & $5 \times 3.7 \times 0.05$ \\
\hline Gauge factor $(\mathrm{GF})$ & $130 \pm 5 \%$ \\
\hline $\mathrm{TCR}\left[1 /{ }^{\circ} \mathrm{C}\right]$ & $<0.35 \%$ \\
\hline $\mathrm{TCGF}\left[1 /{ }^{\circ} \mathrm{C}\right]$ & $<0.28 \%$ \\
\hline Maximum working current $[\mathrm{mA}]$ & 10 \\
\hline Operating temperature range $\left[{ }^{\circ} \mathrm{C}\right]$ & $-50 \sim+80$ \\
\hline Fatigue life time $[$ cycles] & $2 \cdot 10^{6}$ \\
\hline Strain limit $[\mu \varepsilon]$ & 6000 \\
\hline
\end{tabular}

Fig. 6 shows the coordinate system and the location of the sensors on the fork. The $X$ axis is the shaft longitudinal direction and coincides with the wire longitudinal direction in the rest configuration; the $\mathrm{Y}$ axis is the fork longitudinal direction, and the $\mathrm{Z}$ axis is transversal to both elements. Gauges GR2, GR3, GL2 and GL3 are sensitive to deflections in the Z direction ("transversal direction"); gauges GR1 and GL1 are mainly sensitive to the deflections in X direction ("Iongitudinal direction"). In fact gauges GR1 and GL1 are sensitive to the deflections both in the X and Z directions. However, as they are bonded in the neutral bending axis of the $Z$ deflections, even if $Z$ 
deflections do occur, the total strain variation on these strain gauges due to $Z$ deflections are negligible.

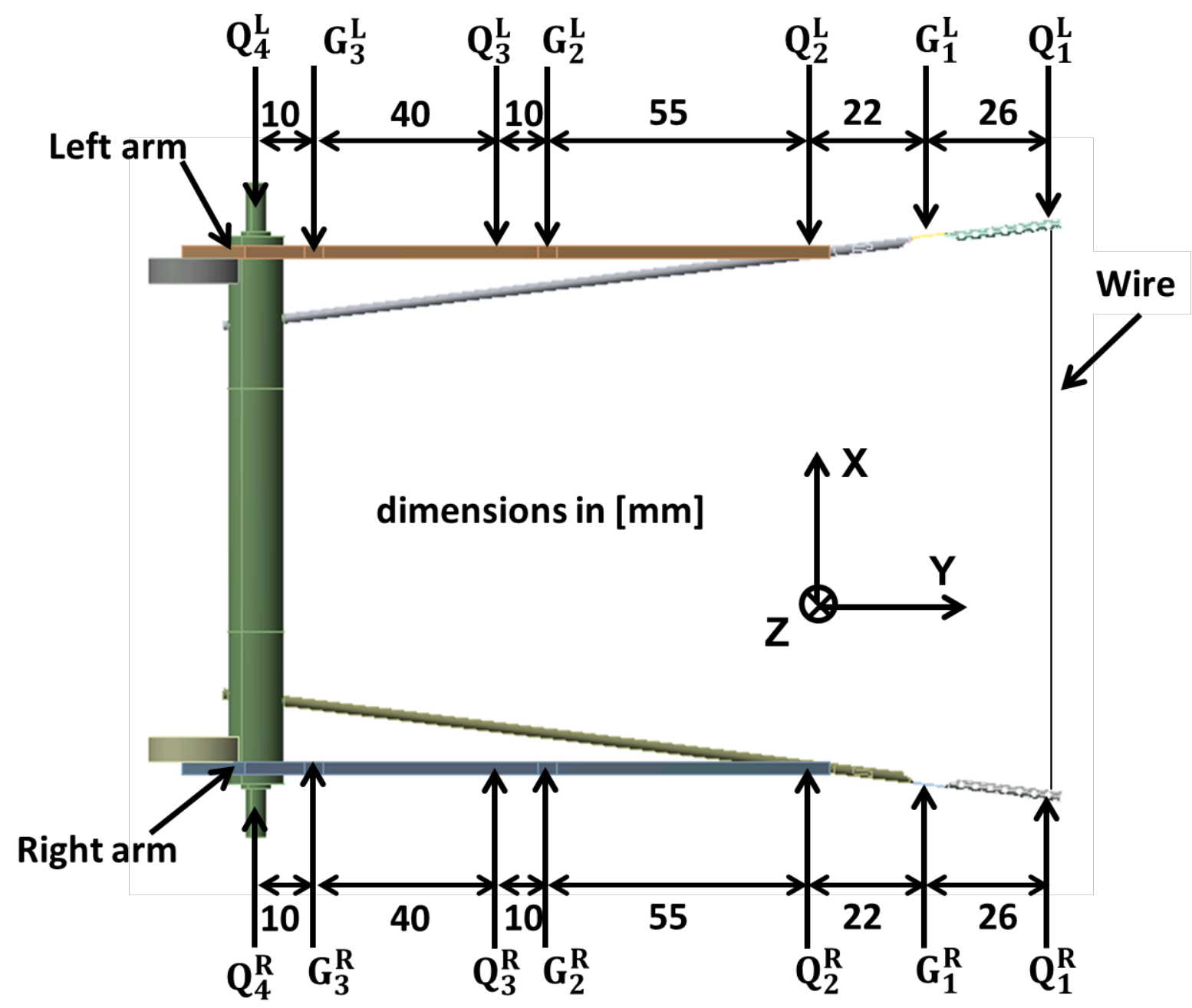

Fig. 6: Location of the semiconductor strain gauges on the $P S$ fork $\left(G_{n}^{R, L}\right)$, location of points whose deflection has been measured $\left(Q_{n}^{R, L}\right)$, and definition of the coordinate system.

An electronic circuit based on a Wheatstone bridge and an amplifier has been developed to measure the strain gauge and wire resistance variations. The amplified tension is read by the oscilloscope. This acquisition system records also the angular position of the fork by means of the actuator angular sensor.

The electronic readout system for the strain gauges consists of four identical circuits. Therefore four strain gauges can be measured at the same time. A similar system has been 
developed for the wire resistance variation. It consists of a single Wheatstone bridge and a single amplifier.

\section{CALIBRATION PROCEDURES}

The final goal of the calibration procedure is to be able to quantify the X-and Z-deflections of the fork arm and the changes of the wire length (wire elongation) $\Delta L$, from the measurement of the electrical tension variations $[\Delta U]$ recorded by the acquisition system during the scan cycle.

Certainly the gauges GRn/GLn record strain mean values in the region of $3.8 \mathrm{~mm}$ where they are located. Those mean values, expressed as electrical tension changes $\Delta U$, will be identified as the values at locations $G_{n}^{R} / G_{n}^{L}$.

Gauges GR1 and GL1 are mounted on the fork flexible hinges (locations $G_{1}^{R}$ and $G_{1}^{L}$ ). Consequently they are mainly sensitive to the wire elongation changes (and so to the X-deflections of the fork). Their calibration should provide the following coefficients to determine fork tip position changes and change of distance between the two tips:

$$
\mathrm{Cx}_{\mathrm{GR} 1}=\frac{\Delta \mathrm{X}\left(\mathrm{Q}_{1}^{\mathrm{R}}\right)}{\Delta \mathrm{U}(\mathrm{GR} 1)}, \mathrm{Cx}_{\mathrm{GL1}}=\frac{\Delta \mathrm{X}\left(\mathrm{Q}_{1}^{\mathrm{L}}\right)}{\Delta \mathrm{U}(\mathrm{GL} 1)} .
$$

Gauges $G R 2$ and $G L 2$ are mounted on the fork middle zone (points $G_{1}^{R}$ and $G_{2}^{L}$ respectively), whereas gauges $G R 3$ and $G L 3$ are mounted on fork zones close to the shaft (points $G_{3}^{R}$ and $G_{3}^{L}$ respectively). The four are mainly sensitive to the Z-transversal deflections of the fork. Their calibration should provide the following coefficients:

$$
\frac{\Delta \mathrm{Z}\left(\mathrm{Q}_{1}^{\mathrm{R}}\right)}{\Delta \mathrm{U}(\mathrm{GR} 2)}, \frac{\Delta \mathrm{Z}\left(\mathrm{Q}_{1}^{\mathrm{R}}\right)}{\Delta \mathrm{U}(\mathrm{GR} 3)}, \frac{\Delta \mathrm{Z}\left(\mathrm{Q}_{1}^{\mathrm{L}}\right)}{\Delta \mathrm{U}(\mathrm{GL} 2)}, \frac{\Delta \mathrm{Z}\left(\mathrm{Q}_{1}^{\mathrm{L}}\right)}{\Delta \mathrm{U}(\mathrm{GL} 3)} .
$$

In general, it is not simple to obtain those calibration coefficients under the real dynamical conditions (the fork deflections and the wire elongation during the scan cycle are generated by the inertia forces associated with the rotation of the shaft). In the present case, less demanding conditions have been adopted and are presented in the following three subsections.

For the sake of simplicity, some calibration coefficients have been obtained through separate static measurements on the fork and on the wire (in other words, those two elements are not assembled together during the calibration process). 
The measurements have to be complemented by a Finite Elements (FE) simulation of the fork deflections. This FE model will also be used to assess the influence of the dynamic conditions (associated with the fork rotation during the scan cycle) on the calibration coefficients. The model will have to be tuned to properly match the measurements.

\subsection{Calibration coefficient for the wire elongation}

Measuring the wire resistance elongation coefficient is done by recording the resistance variation $(\Delta R)$ as a function of the elongation changes $(\Delta L)$. The procedure has consisted on increasing the wire length progressively and measuring simultaneously the resistance variation. Fig. 7 shows the experimental setup, which includes a $10 \mu \mathrm{m}$ resolution manual linear stage equipped with an electrically isolated clamp, a fixed point with another clamp (each wire end is attached to a clamp), and two electrical cables to connect the multimeter.

The gauge factor (GF) can be determined through $G F=\left(\Delta R / R_{0}\right) /\left(\Delta L / L_{0}\right)$, where $R_{0}$ and $L_{0}$ are the initial resistance and length of the wire respectively. The wire tension elongation coefficient $\left(C L_{w}\right)$ has been obtained using the same experimental setup, however the multimeter has been replaced by the Wheatstone bridge circuit in order to measure the wire tension variations $(\Delta U)$. Fig. 8 shows the measured curve for the case of the $34 \mu \mathrm{m}$ diameter carbon wire typically mounted on the fork tips.

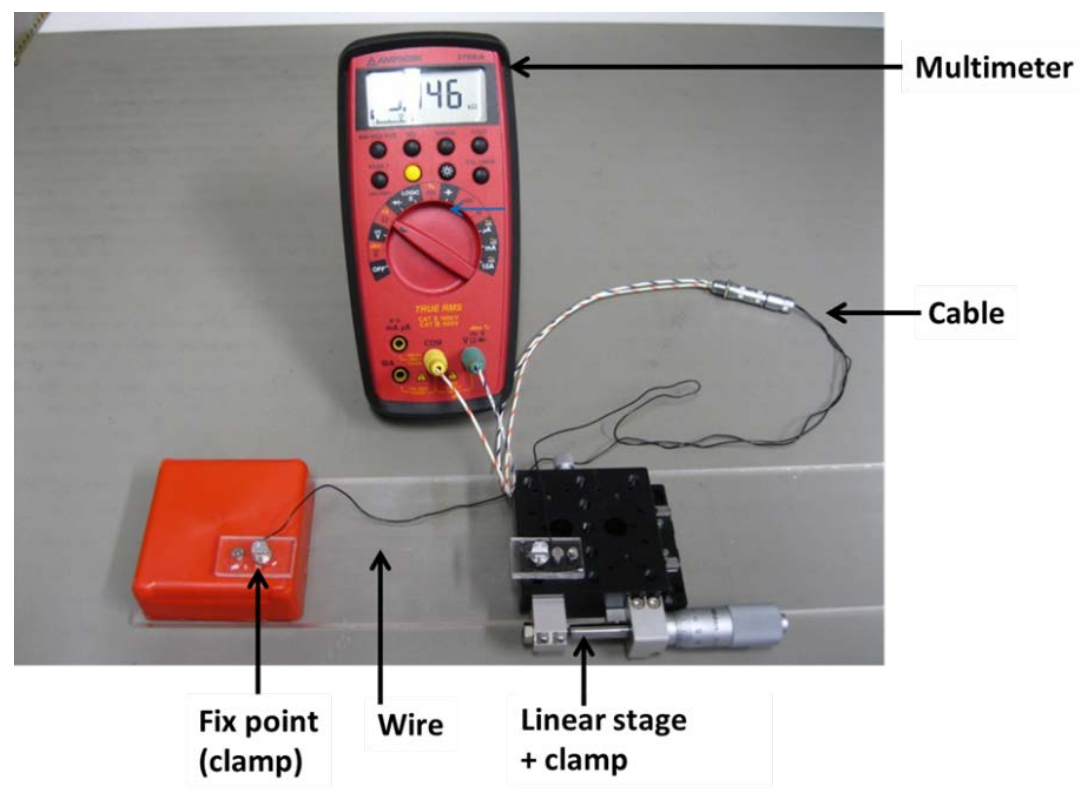

Fig. 7: Experimental setup used to measure wire resistance variation as a function of the wire elongation. 


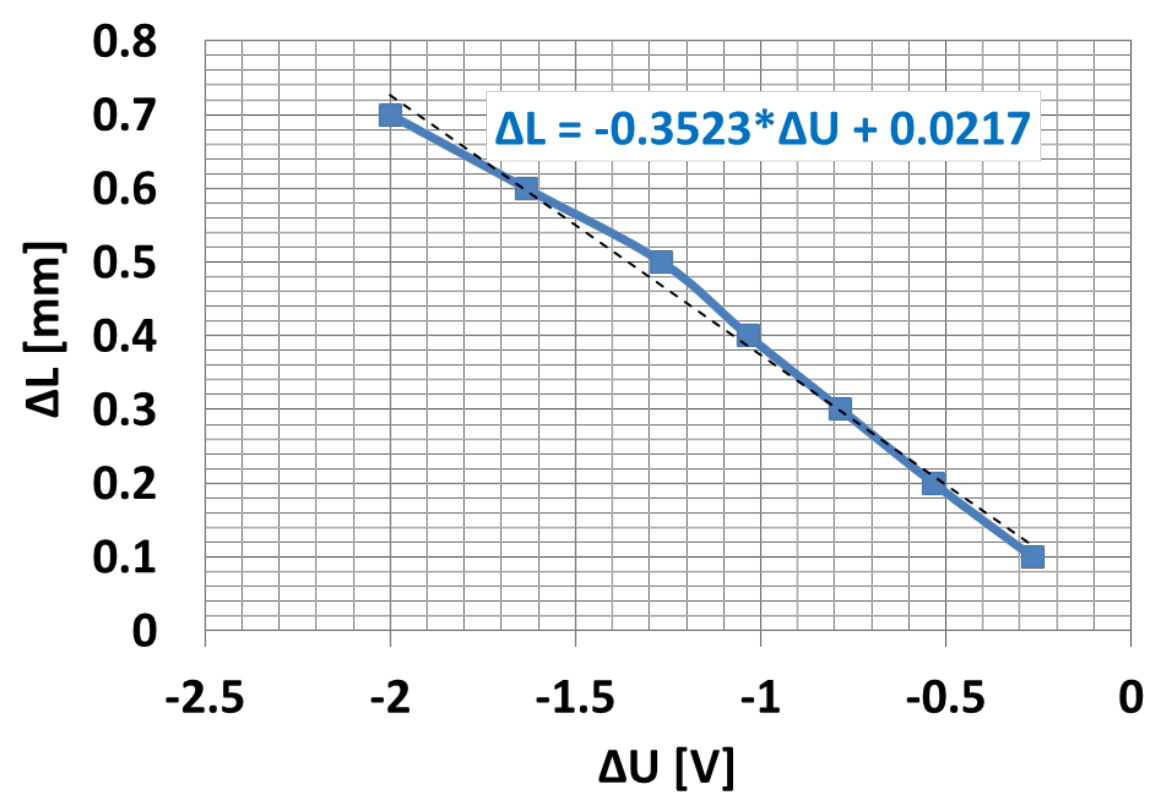

Fig. 8: Change of wire elongation as function of voltage change.

A second setup consisting in a calibrated dynamometer, a calliper (to measure the wire elongation) and a fixed point has been used to determine the wire axial stiffness $k$ through $k=F /\left(L-L_{0}\right)$, where $F$ is the force measured by the dynamometer.

Table 2 summarizes the main characteristics of the carbon wire and the result of the calibration.

Table 2: Wire characteristics and calibration coefficients

\begin{tabular}{|l|l|l|l|}
\hline$\varnothing$ & Wire diameter & 34 & {$[\mu \mathrm{m}]$} \\
\hline$\rho$ & Density & 1800 & {$\left[\mathrm{~kg} / \mathrm{m}^{3}\right]$} \\
\hline $\mathrm{L}_{0}$ & Wire length & 115 & {$[\mathrm{~mm}]$} \\
\hline $\mathrm{k}$ & Axial stiffness & 340 & {$[\mathrm{~N} / \mathrm{m}]$} \\
\hline$F_{\max }$ & Breaking force & 0.5 & {$[\mathrm{~N}]$} \\
\hline $\mathrm{R}$ & Resistance & 31 & {$[\mathrm{Ohm} / \mathrm{mm}]$} \\
\hline $\mathrm{CL} \mathrm{W}_{\mathrm{W}}$ & Tension elongation coefficient & -0.35 & {$[\mathrm{~mm} / \mathrm{V}]$} \\
\hline $\mathrm{GF}$ & Gauge factor & 0.64 & \\
\hline
\end{tabular}




\subsection{Calibration coefficients for the fork X-deflections}

The calibration of gauges GR1 and GL1 (sensitive to the $X$ deflections) has been achieved through a simple procedure consisting in imposing controlled progressive deflections on the fork tips in the $\mathrm{X}$ direction $\left(\Delta \mathrm{X}\left(\mathrm{Q}_{1}^{\mathrm{R}}\right), \Delta \mathrm{X}\left(\mathrm{Q}_{1}^{\mathrm{L}}\right)\right)$ and recording simultaneously the tension changes $(\Delta \mathrm{U}(\mathrm{GR} 1), \Delta \mathrm{U}(\mathrm{GL1}))$ using the Wheatstone bridge circuit.

Fig. 9 shows two typical curves (for the left and right fork). Their slope represents the calibration coefficient. Table 3 summarizes the results.

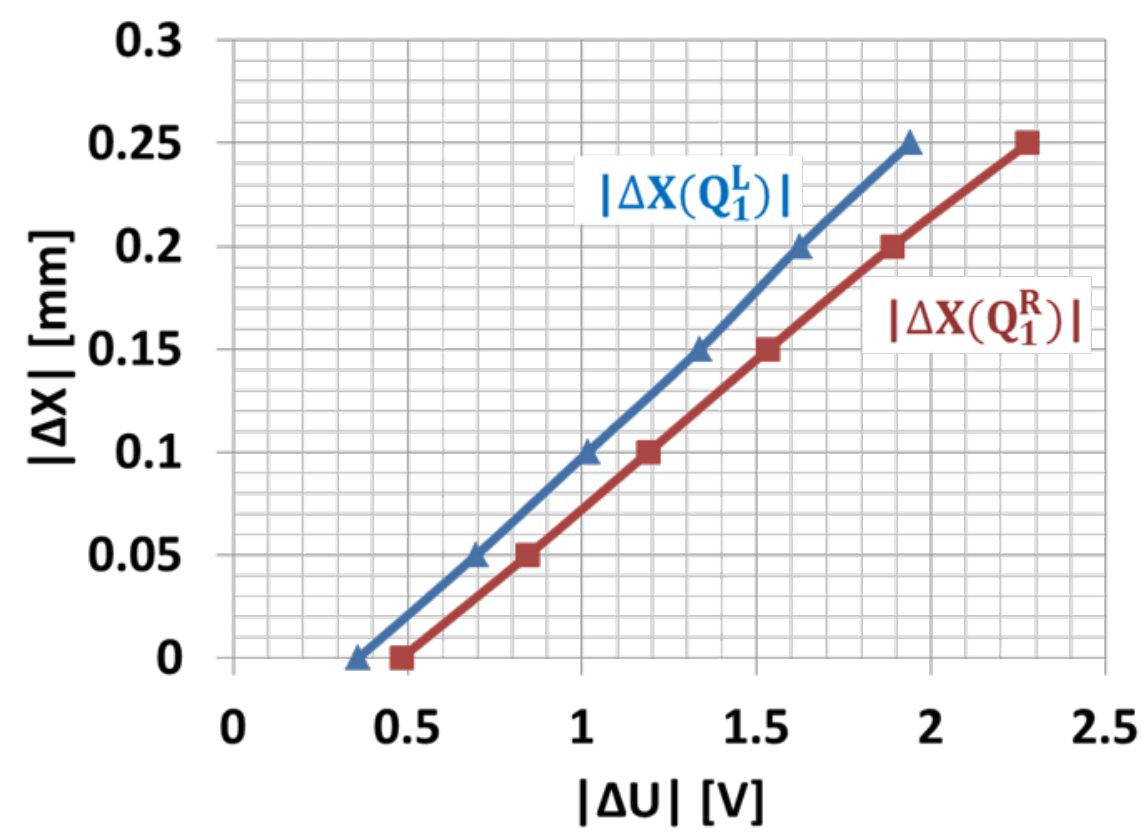

Fig. 9: Typical result of fork tip deflections $\Delta X\left(Q_{1}^{R}\right)$ and $\Delta X\left(Q_{1}^{L}\right)$ vs. tension variations on $G L 1$ and GR1, respectively.

Table 3: X-deflection calibration coefficients

\begin{tabular}{|c|c|}
\hline Coefficient & {$[\mathrm{mm} / \mathrm{V}]$} \\
\hline $\mathrm{CX}_{\mathrm{GR} 1}$ & 0.140 \\
\hline $\mathrm{CX}_{\mathrm{GL} 1}$ & -0.158 \\
\hline
\end{tabular}

\subsection{Calibration coefficients for the fork Z-deflections}

This calibration is also based on static measurements but, unlike the preceding ones, the possible influence of the dynamical conditions on the deflection has been taken into account 
through Finite Elements (FE) simulations. The whole procedure can be described as a hybrid one and can be split into four steps:

- Calibration of the strain gauges under static loads.

- FE simulation of the fork under static loads.

- FE simulation of the fork under dynamic loads.

- Correlation of the previous results.

\section{Calibration of the Z-strain gauges under static loads}

For the static calibration, the fork is mounted on a rigid and stable support (in the same configuration as when mounted on the actuator) and subjected to a progressive load $P_{\exp }^{\text {st }}$ on a point $Q\left(Q_{R}\right.$ and $Q_{L}$ for the right and left fork arms respectively) close to the fork tips (Fig. 10). For each particular load, the tension variations $\left(\Delta U_{\exp }^{\text {st }}(G R n), \Delta U_{\exp }^{\text {st }}(G L n)\right)$ in the strain gauges sensitive to the Z-deflections $(n=2,3)$ are recorded together with the fork deflections $z_{\exp }^{\text {st }}$ at the $\mathrm{Q}_{2}^{\mathrm{R}, \mathrm{L}}$ and $\mathrm{Q}_{3}^{\mathrm{R}, \mathrm{L}}$ locations by means of dial comparators.

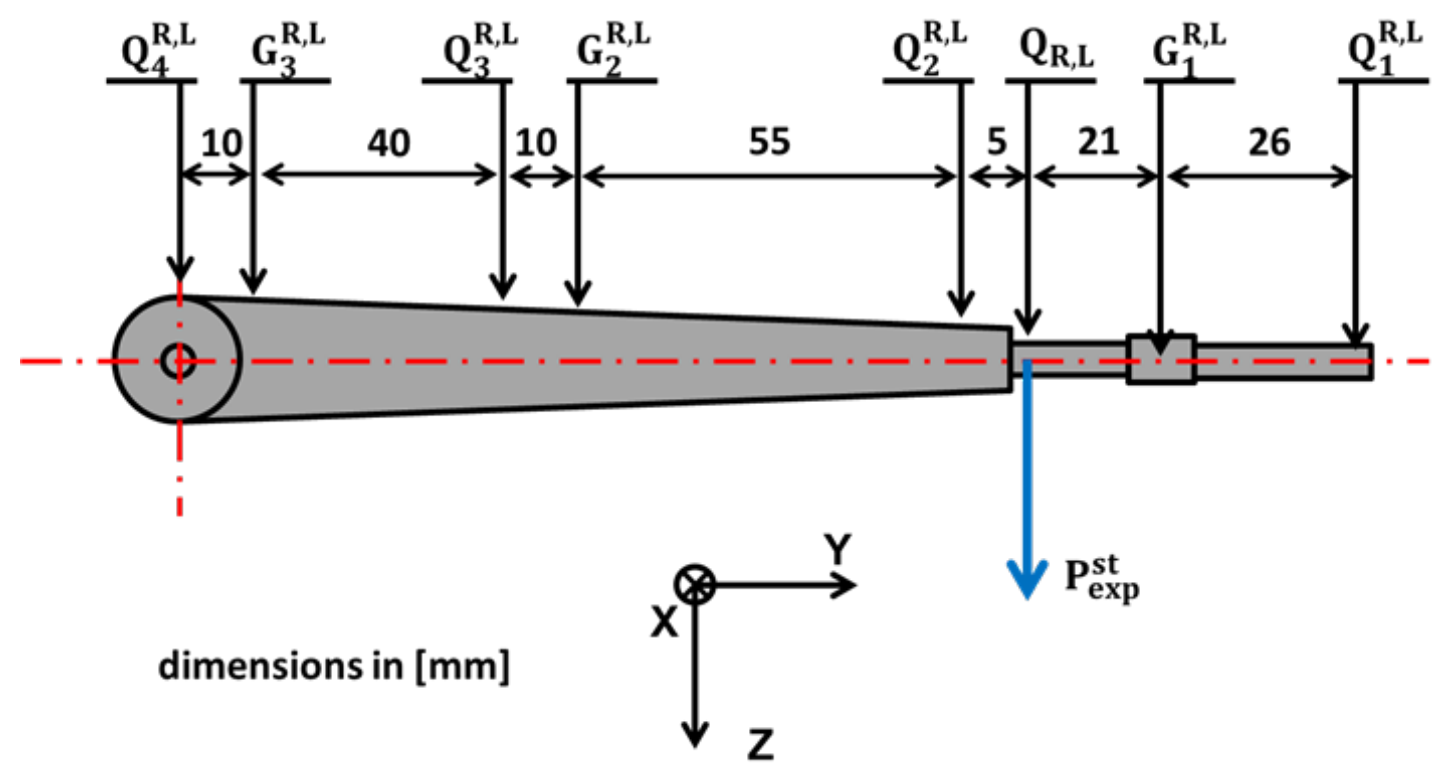

Fig. 10: PS fork and applied load at $Q_{R, L}$ locations. $Q_{n}^{R, L}$ and $G_{n}^{R, L}$ are the measurement points and the gauge locations (as in Fig. 6). 
The correlation coefficient between the applied loads and the strain gauge tension variations and that between load and fork deflection is thus obtained for each location:

$$
\frac{\Delta U_{\exp }^{s t}(G R n)}{P_{\exp }^{s t}\left(Q_{L}\right)}, \frac{z_{\exp }^{s t}\left(Q_{n}^{R}\right)}{P_{\exp }^{s t}\left(Q_{L}\right)}, \frac{\Delta U_{\exp }^{s t}(G L n)}{P_{\exp }^{s t}\left(Q_{R}\right)}, \frac{z_{\exp }^{s t}\left(Q_{n}^{L}\right)}{P_{\exp }^{s t}\left(Q_{L}\right)}, n=2,3 .
$$

\section{FE static and dynamic simulations of the fork}

The same conditions as described in the previous paragraphs (boundary conditions, applied loads) have been used to simulate the static fork deflections $Z_{\mathrm{FE}}^{\mathrm{st}}$ and the strain values $\varepsilon_{\mathrm{FE}}^{\mathrm{st}}$ at points $G_{2}^{R, L}$ and $G_{3}^{R, L}$ through a FE model.

In the FE model (Fig. 11) the wire was modelled by an analytical spring (preloaded with a tension of $0.3 \mathrm{~N}$ ) tensioned between the two tips with the same axial stiffness as the real wire, $k=$ $340 \mathrm{~N} / \mathrm{m}$. The mass of wire soldering spots has been introduced as two mass points analytically modelled and located on the fork tips, with value $m=0.06$ grs.

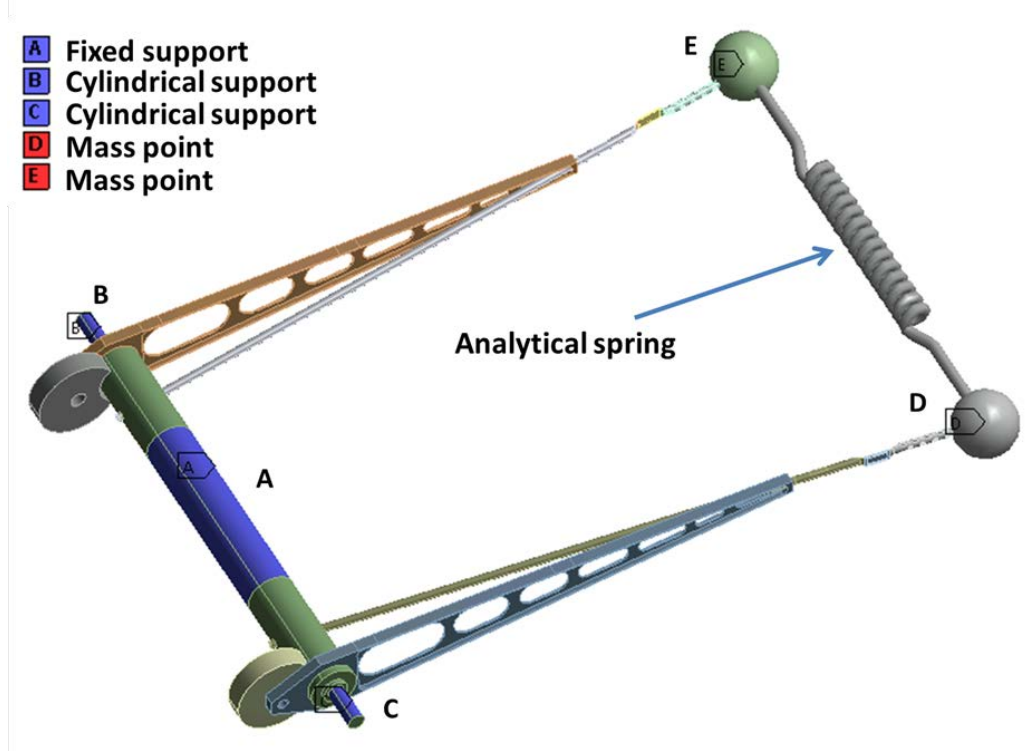

Fig. 11: Finite Element model of the fork wire arrangement.

Fig. 12 shows the results of the displacement at locations $Q_{2}^{R, L}$. As the FE model is perfectly symmetrical, $z_{F E}^{s t}\left(Q_{2}^{R}\right)=Z_{F E}^{s t}\left(Q_{2}^{L}\right)$. Of course this is not the case for the real system, and consequently the measured Z-deflections are not equal: $z_{\exp }^{s t}\left(Q_{2}^{R}\right) \neq z_{\exp }^{s t}\left(Q_{2}^{L}\right)$. 


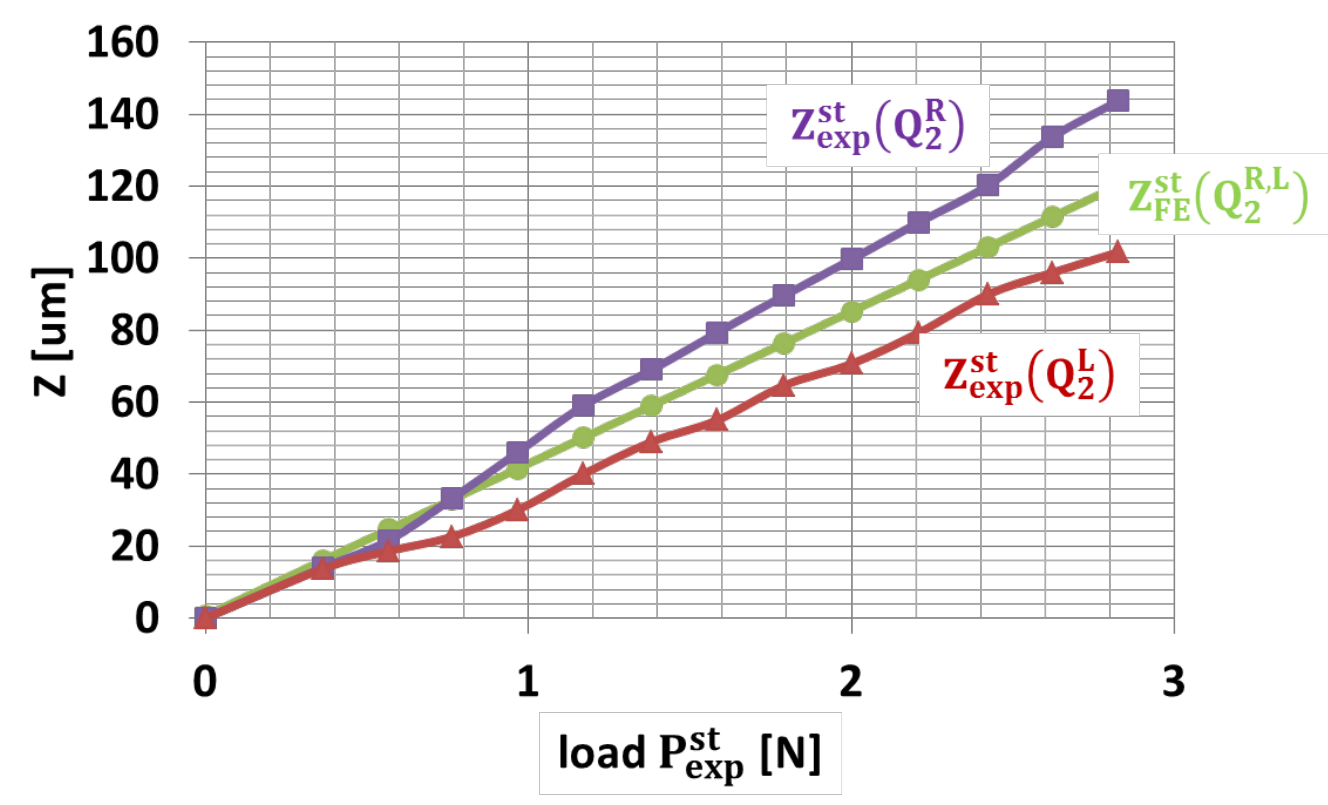

Fig. 12: Measured (exp) and calculated (FE) static deflections at locations $Q_{2}^{R, L}$.

The correlation coefficients between loads and the resulting strains at points $G_{2}^{R, L}$ and $G_{3}^{R, L}$ are readily obtained based on the measurements:

$$
\frac{\varepsilon_{F E}^{s t}\left(G_{n}^{R}\right)}{P_{\exp }^{s t}\left(Q_{L}\right)}, \frac{\varepsilon_{F E}^{s t}\left(G_{n}^{L}\right)}{P_{\exp }^{\text {st }}\left(Q_{R}\right)}, n=2,3 .
$$

Finally, the fork has been subjected to different values of angular acceleration $\ddot{\theta}$ (from 0 to $\left.12000 \mathrm{rad} / \mathrm{s}^{2}\right)$, and the corresponding deflections of the fork tips $Z_{\mathrm{FE}}^{\text {dyn }}\left(Q_{1}^{R}, \ddot{\theta}\right), z_{\mathrm{FE}}^{\text {dyn }}\left(Q_{1}^{L}, \ddot{\theta}\right)$ and the strain values $\varepsilon_{\mathrm{FE}}^{\text {dyn }}\left(G_{2}^{R, L}, \ddot{\theta}\right), \varepsilon_{\mathrm{FE}}^{\text {dyn }}\left(G_{3}^{\mathrm{R}, \mathrm{L}}, \ddot{\theta}\right)$ have been calculated through the $\mathrm{FE}$ analysis (Fig. 13). The corresponding correlation coefficients are:

$$
\frac{z_{F E}^{d y n}\left(Q_{1}^{R}, \ddot{\theta}\right)}{\varepsilon_{F E}^{d y n}\left(G_{n}^{R}, \ddot{\theta}\right)}, \frac{z_{F E}^{d y n}\left(Q_{1}^{L}, \ddot{\theta}\right)}{\varepsilon_{F E}^{d y n}\left(G_{n}^{L}, \ddot{\theta}\right)}, n=2,3 .
$$




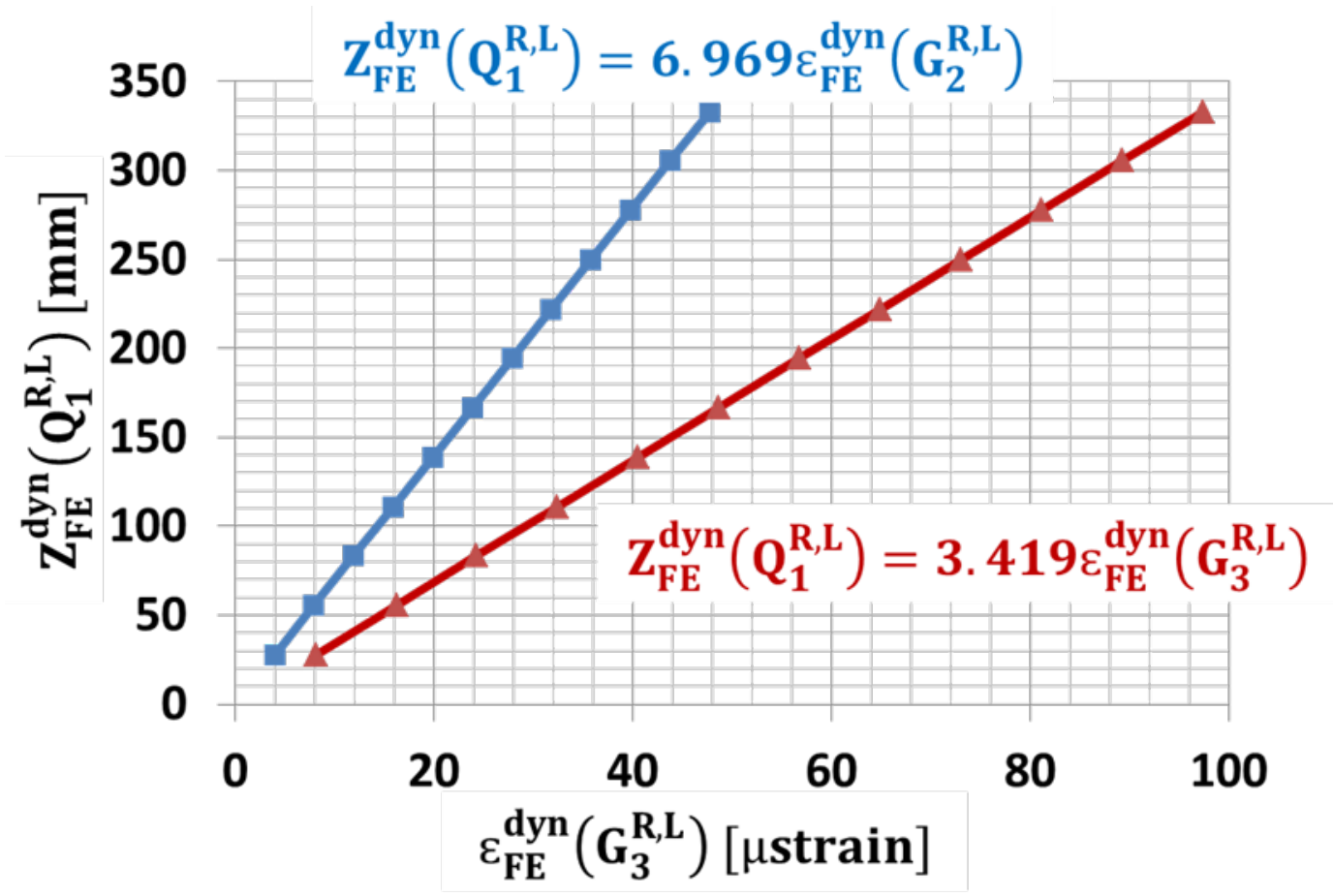

Fig. 13: Typical result of calculated fork tip deflection vs. calculated strain for values of angular acceleration in the range $(0-12000) \mathrm{rad} / \mathrm{s}^{2}$.

Comparisons between the FE results and the measurements yield the calibration coefficients relating the Z-deflection of the fork tips under dynamic conditions, $z_{F E}^{d y n}\left(Q_{1}^{R, L}, \ddot{\theta}\right)$, and the electrical tension variation recorded by the Z-strain gauges (also under dynamic conditions):

$$
\frac{z_{F E}^{\text {dyn }}\left(Q_{1}^{R}, \ddot{\theta}\right)}{\Delta U_{\exp }^{\text {dyn }}\left(G_{n}^{R}, \ddot{\theta}\right)}, \quad \frac{z_{F E}^{\text {dyn }}\left(Q_{1}^{L}, \ddot{\theta}\right)}{\Delta U_{\exp }^{\text {dyn }}\left(G_{n}^{L}, \ddot{\theta}\right)}, n=2,3 .
$$

An example is given in Fig. 14. The calibration procedure is summarized in Fig. 15, and the detailed results are shown in Table 4. 


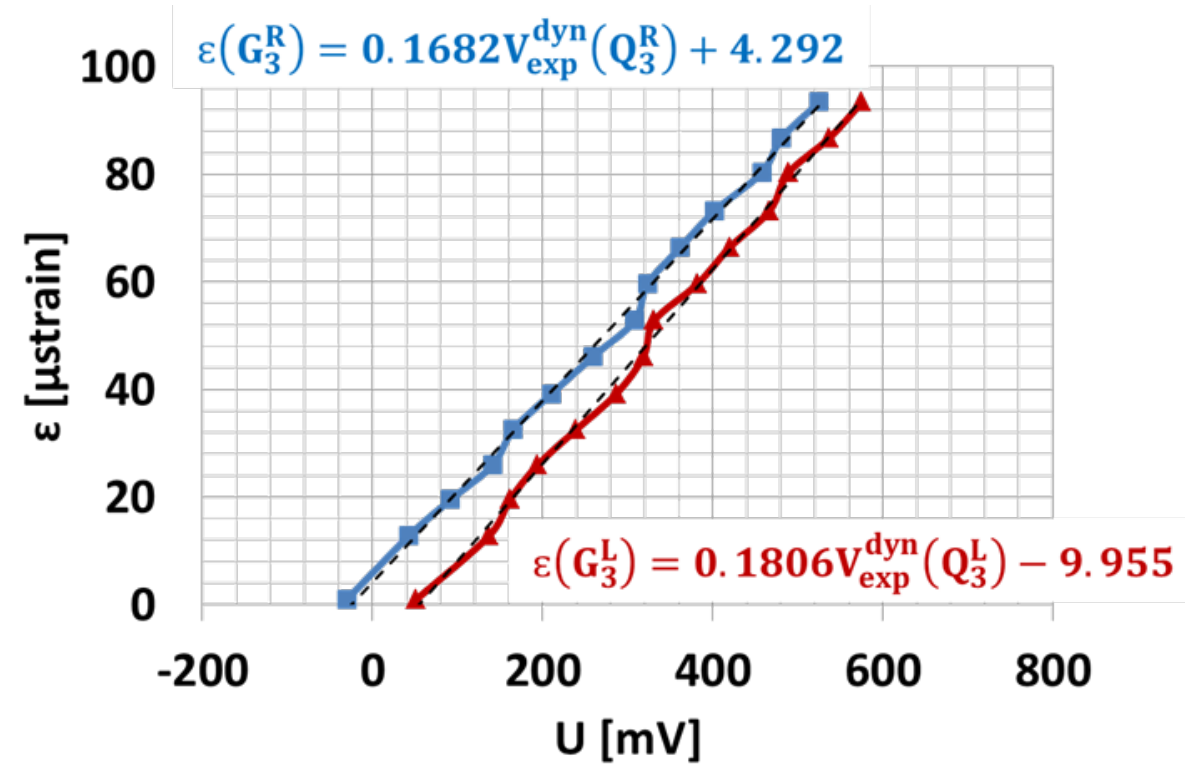

Fig. 14: Typical result of correlation between the tension recorded by gauges GR3 and GL3, and the strain at points $G_{3}^{R, L}$ calculated with the FE model.

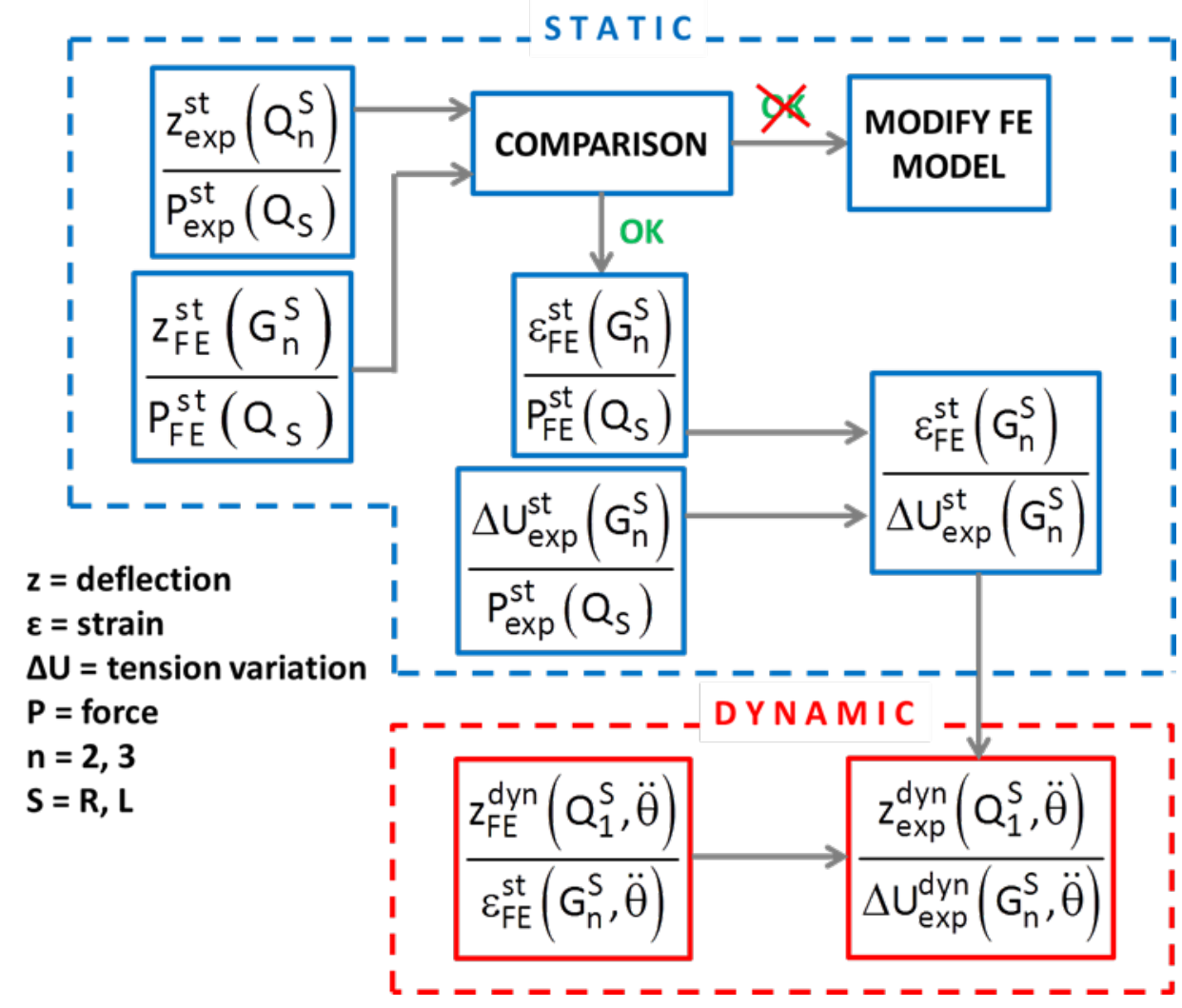

Fig. 15: Flow diagram showing the calibration procedure. The diagram is subdivided in a static part (measurements and FE) and a dynamic part (only FE based). 
Table 4: Z-deflection dynamic calibration coefficients

\begin{tabular}{|c|c|}
\hline Coefficient & $\begin{array}{c}\mathrm{z}_{\mathrm{FE}}^{\text {dyn }}(\ddot{\theta}) / \Delta \mathrm{U}_{\exp }^{\text {dyn }}(\ddot{\theta}) \\
{[\mathrm{mm} / \mathrm{mV}]}\end{array}$ \\
\hline $\mathrm{CZ}_{\mathrm{GR2}}$ & 0.001329 \\
\hline $\mathrm{CZ}_{\mathrm{GR3}}$ & 0.000652 \\
\hline $\mathrm{CZ}_{\mathrm{GL} 2}$ & 0.001329 \\
\hline $\mathrm{CZ}_{\mathrm{GL3}}$ & 0.000652 \\
\hline
\end{tabular}

\section{VIBRATION MEASUREMENTS}

Fig. 16 shows a schematic drawing of the three variables that have been measured in dynamic conditions (during a whole scan cycle): fork X and Z deflections of the fork ("longitudinal" and "transversal" deflections, respectively) and wire elongation $\Delta \mathrm{L}$. As explained in the previous section, the fork longitudinal deflections and the wire elongation are computed from the records of the GR1 and GL1 gauges. The calculation of the fork transversal deflections is done from the records of the GR2 and GR3 gauges for one arm, and of the GL2 and GL3 gauges for the other one.

The scan cycle consists on a rotational motion of the fork and wire, and can be divided into three phases:

- Motion from OUT to IN: the wire intersects the beam for the first time on the cycle. The main constraint is the wire speed at the beam crossing instant.

- Waiting time at IN position: both fork and wire are retained at the IN position during a certain time, long compared with the cycle duration.

- Motion from IN to OUT: similar to the first phase but in opposite direction; the wire intersects a second time the beam. Again the main kinematic constraint is given by the required minimum wire speed.

The minimum speed requirement is given by the maximum heat deposition of the beam in the wire in order to avoid fast ageing (high temperature provokes wire material sublimation) and subsequent break due to the inertial forces or, in an extreme situation, an instantaneous sublimation of part of the wire.

Typical motion patterns of the OUT-IN and IN-OUT phases as function of time are shown in Fig. 17. As every scan cycle has to follow the same motion pattern, a unique predefined motion pattern can be used as the reference motion to be followed by the scanner actuator. This 
predefined motion pattern has to be designed in such a way that it matches the requirements of the scan cycle. Requirements concern the full travel length, the beam crossing region and the wire speed at the beam crossing region.
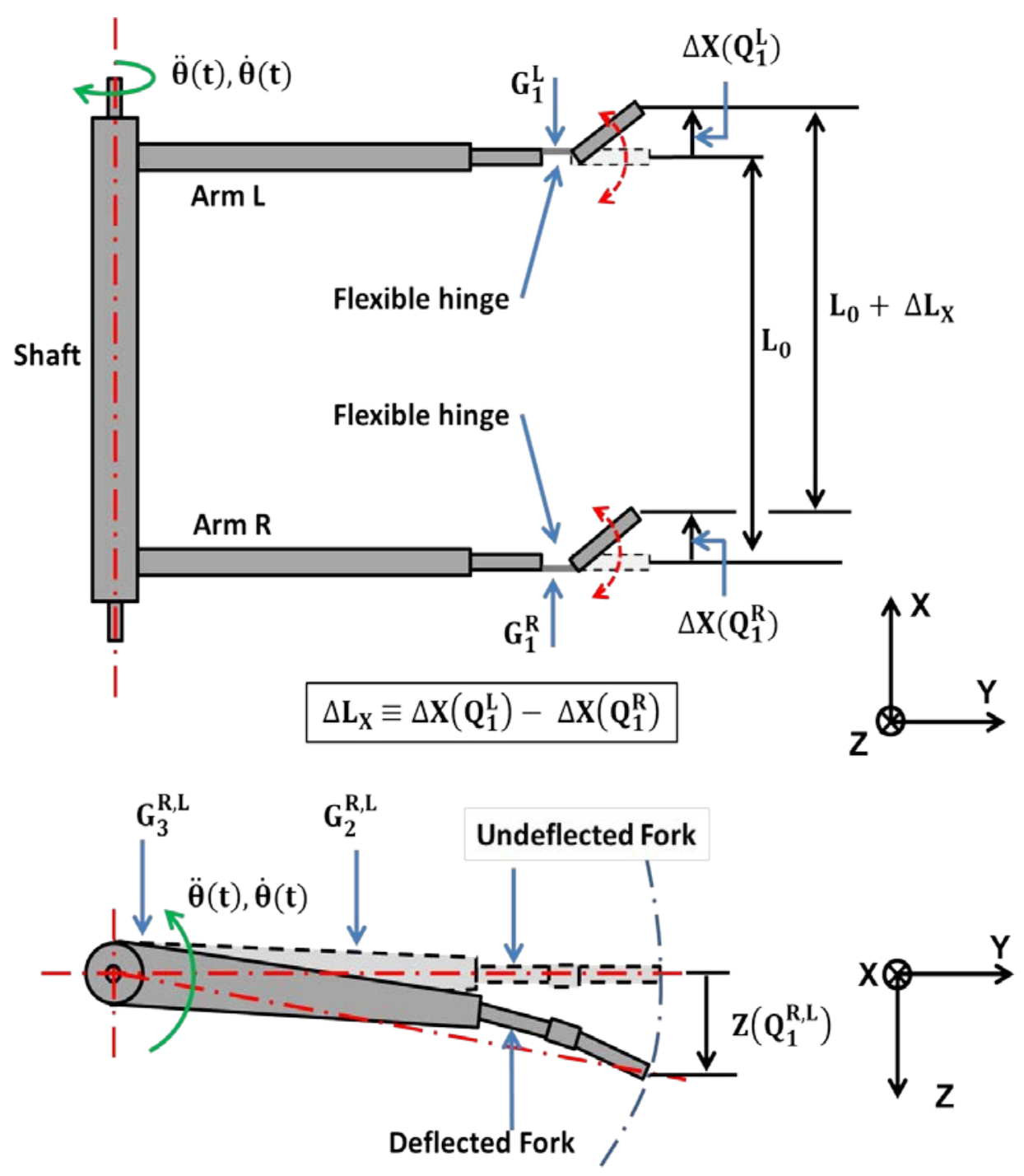

Fig. 16: Schematic of the fork deflections with resulting wire elongation and location of the strain gauges.

Depending on the required peak speed, the fork length, the angular travel and the motion pattern design, the peak acceleration values can vary. Typical values for the PS CERN vacuum wire scanner are shown in Table 5. 
Table 5: Performances of the PS CERN vacuum wire scanner.

\begin{tabular}{|l|l|}
\hline Peak speed [m/s] & 16.4 \\
\hline Fork length [mm] & 164 \\
\hline Peak angular speed [rad/s] & 100 \\
\hline Peak angular acceleration $\left[\mathrm{rad} / \mathrm{s}^{2}\right]$ & 12700 \\
\hline Peak tangential acceleration $[\mathrm{g}]$ & 212 \\
\hline Peak normal acceleration $[\mathrm{g}]$ & 203 \\
\hline
\end{tabular}

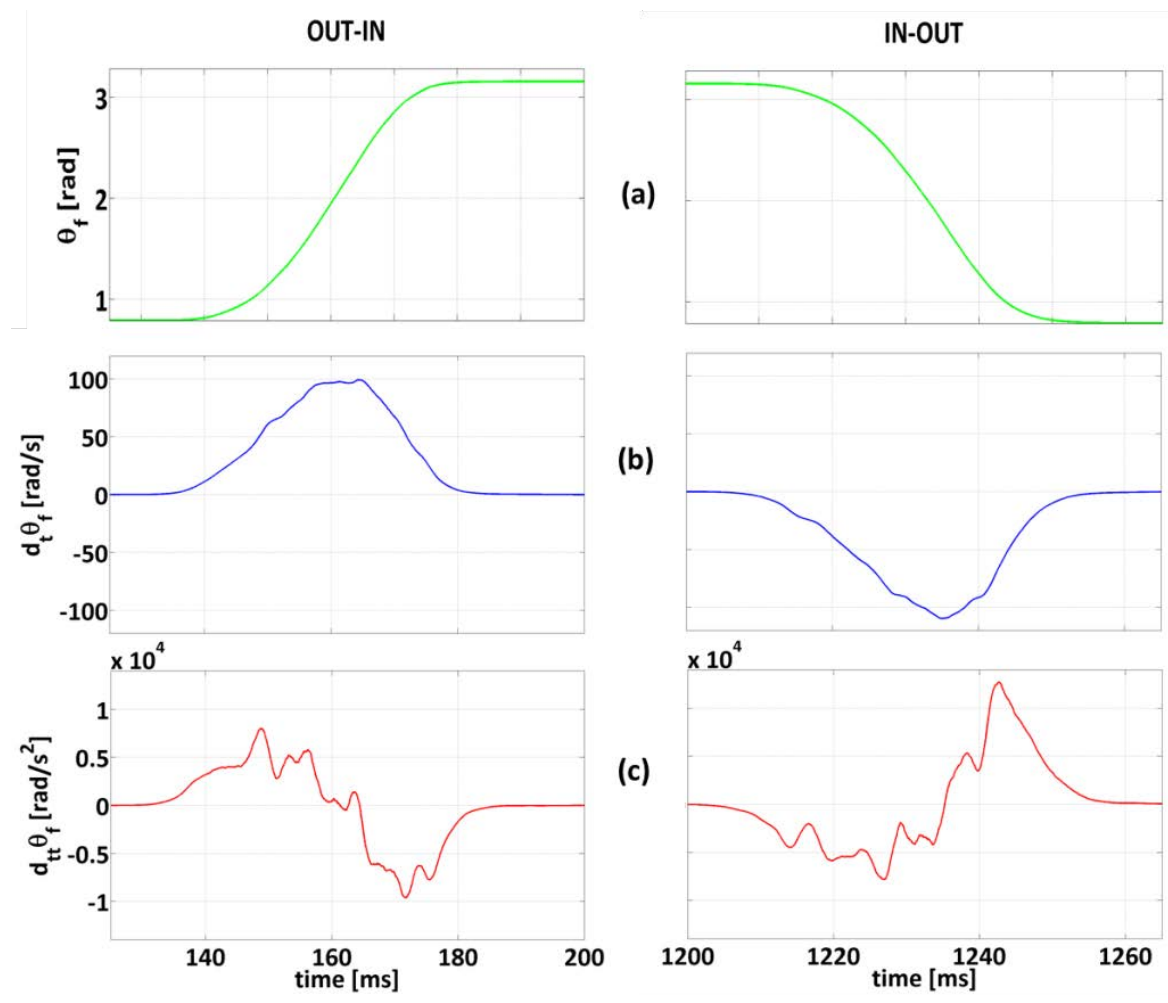

Fig. 17: Typical measured motion patterns for a full scan motion cycle: angular position (a), angular velocity (b) and angular acceleration (c).

\section{X-deflections}

Fig. 18 shows the $\mathrm{X}$-deflections of the fork tips, $\Delta x\left(Q_{1}^{R, L}\right)$, calculated from the measured tension variations $\Delta U_{\exp }^{\text {dyn }}$ at gauges GR1 and GL1 and the corresponding calibration coefficients.

The curves show that as far as vibrations are concerned, the X-deflection behaviour is rather similar for both tips though with phase differences. Both the OUT-to-IN and the IN-to-OUT motion 
show an oscillation of the fork tips with almost the same frequency with opposition phase. However both curves show an offset between OUT and IN position (around -70 $\mu$ m for QL1 and $40 \mu \mathrm{m}$ for QR1). Several tests have been performed in order to find the origin of the difference between the tip offsets between OUT and IN fork positions. Among others, a test consisting on a very gentle manual rotation from OUT to IN has been performed, the wire, the strain gauges signals and potentiometer position (see Fig. 19) have been recorded along this manual motion. The results match the deformation problem on the fork which is schematically shown in Fig. 20. Due to this deformation (defect), the fork tips tend to separate from each other. However, as they are connected through the wire, this results in an increasing on the bending of the hinges, which is measured by the gauges as if were a tip separation. Because of the pulling effect, the wire shows an effect of elongation.
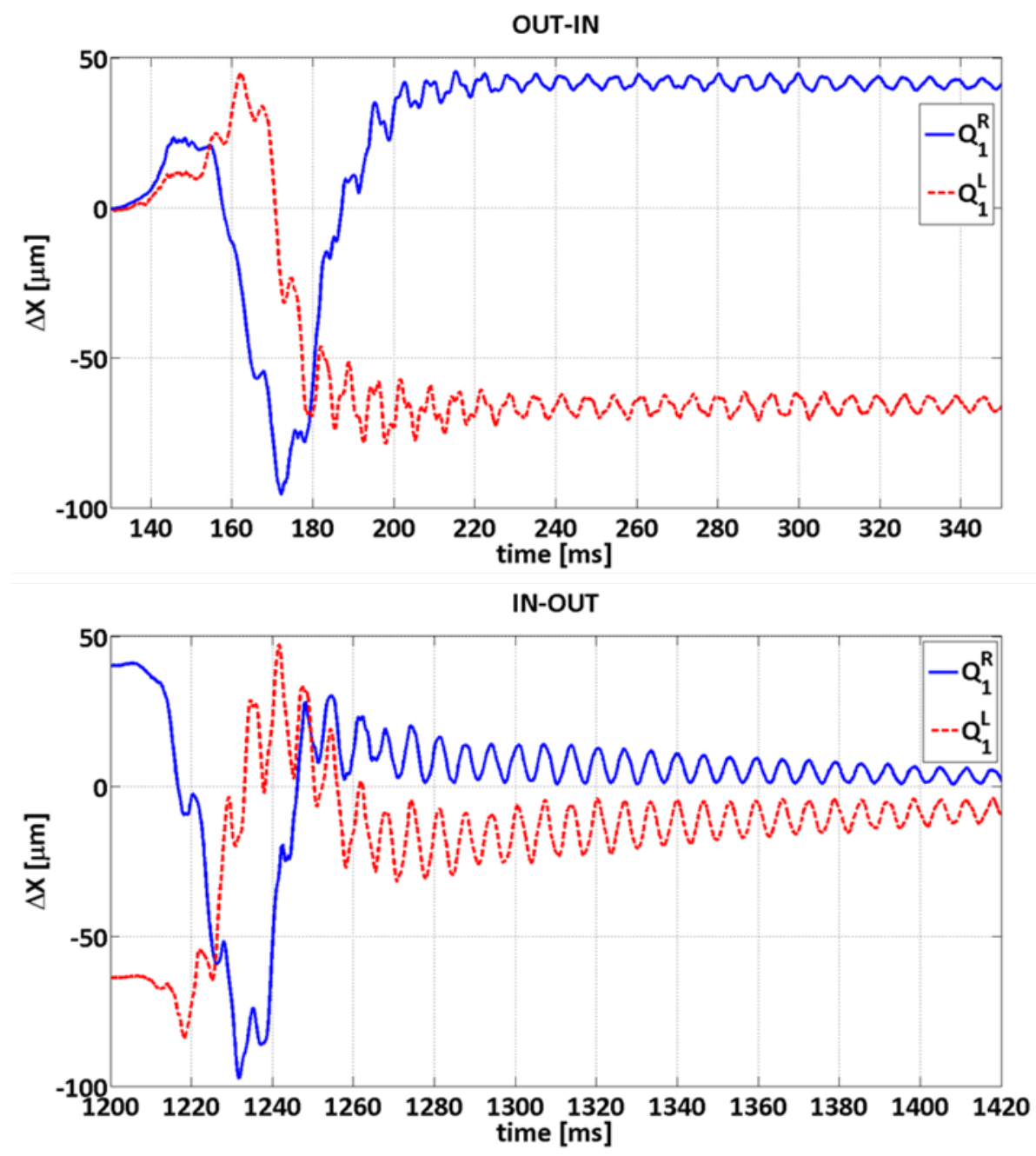

Fig. 18: X-deflection of the fork tips (locations $Q_{1}^{R, L}$ ) for the OUT-IN (top) and the IN-OUT (bottom) phases, calculated from the tension variations at gauges GR1 and GL2. 


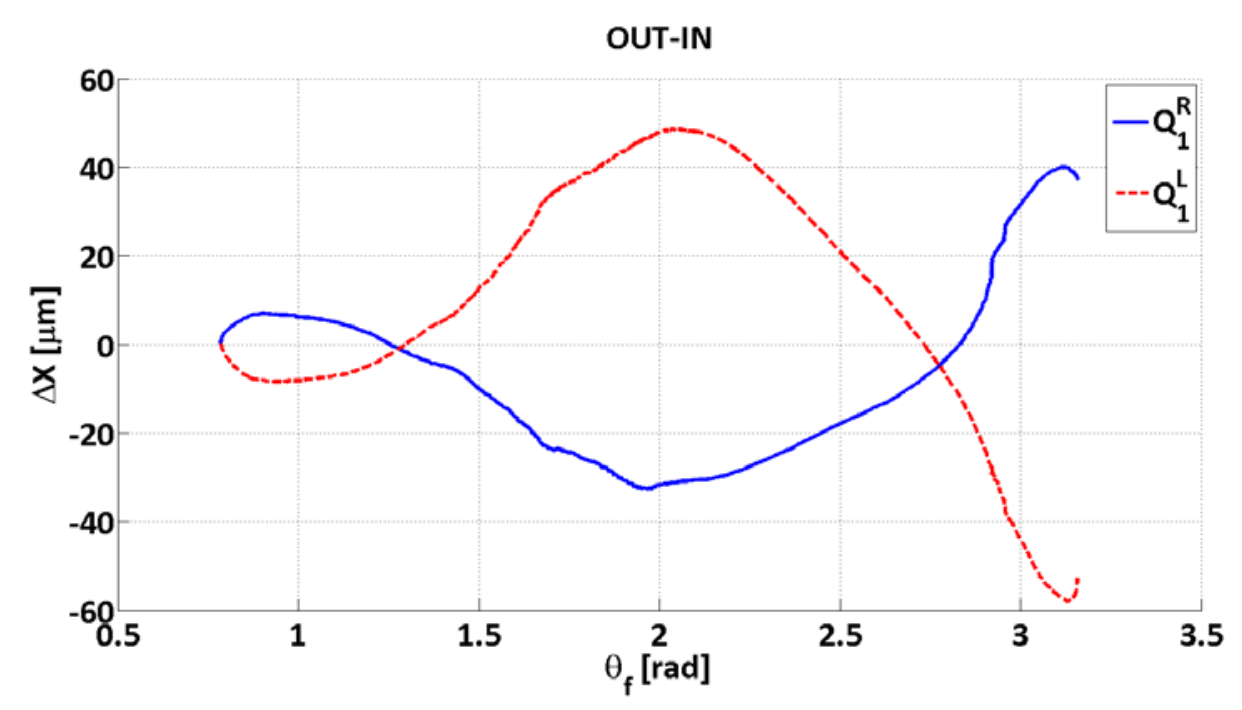

Fig. 19: Values of the tip deflection for a gentle manual rotation.

If the wire did not undergo any transverse deformation, its elongation would be given by $\Delta \mathrm{L}_{\mathrm{x}}=\Delta \mathrm{x}\left(\mathrm{Q}_{1}^{\mathrm{L}}\right)-\Delta \mathrm{x}\left(\mathrm{Q}_{1}^{\mathrm{R}}\right)$, the fork tips separation variation.
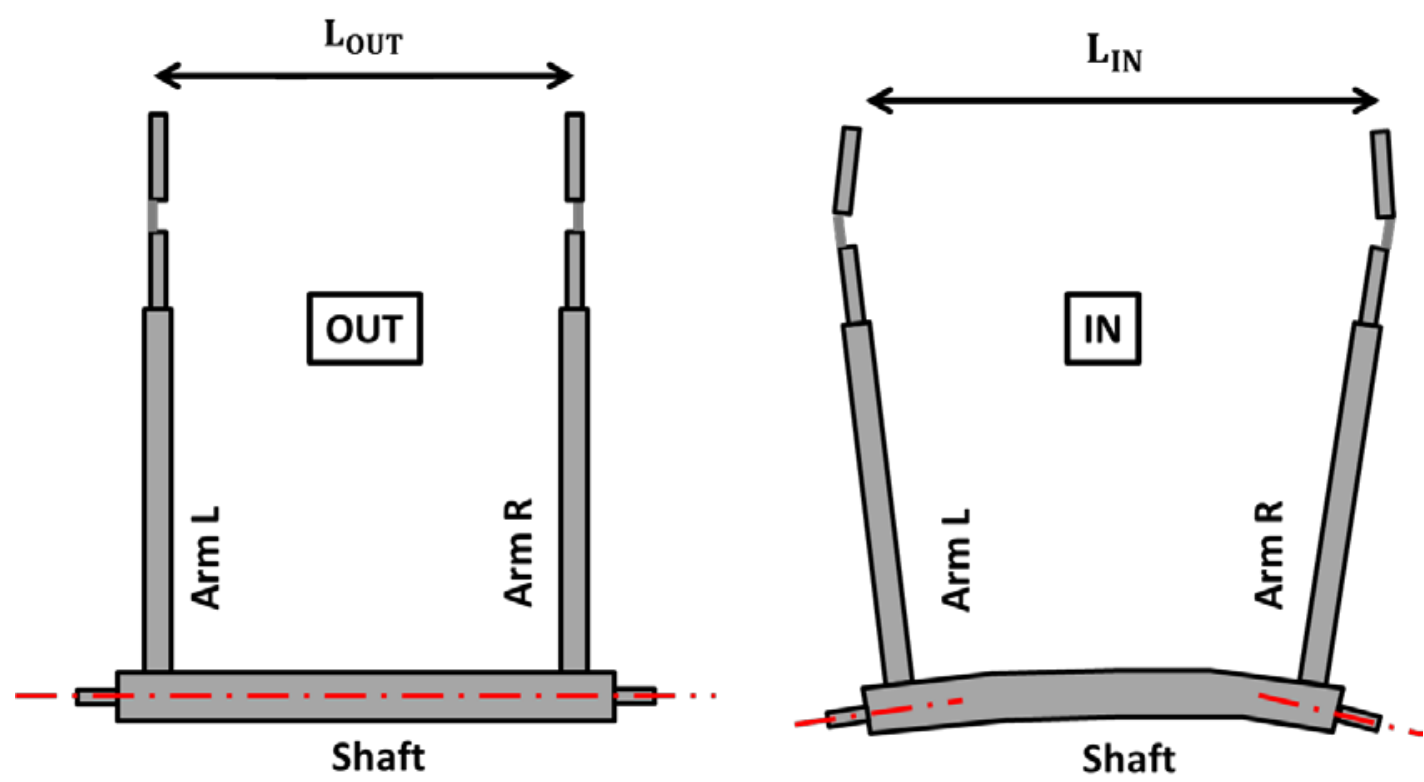

$$
\mathbf{L}_{\text {OUT }}<\mathbf{L}_{\text {IN }}
$$

Fig. 20: Schematics of the phenomenon causing the offset signals between OUT and IN. 


\section{Wire elongation}

Fig. 21 compares the wire elongation $\Delta \mathrm{L}$ assessed from the tension variations due to its resistance and the fork tips separation change $\Delta \mathrm{L}_{\mathrm{x}}$. Suppressing the $\Delta \mathrm{L}$ oscillations with appropriate running sum value, $\Delta \mathrm{L}$ and $\Delta \mathrm{L}_{x}$ show the same trend up to $\mathrm{t}=170 \mathrm{~ms}$ (OUT to IN) and up to $\mathrm{t}=1235 \mathrm{~ms}$ (IN to OUT), $\Delta \mathrm{L}$ shows high oscillations until the end of the motion phase while the tips separation does not. Clearly, then, the wire elongation is not just the "passive" result of the fork tips motion.
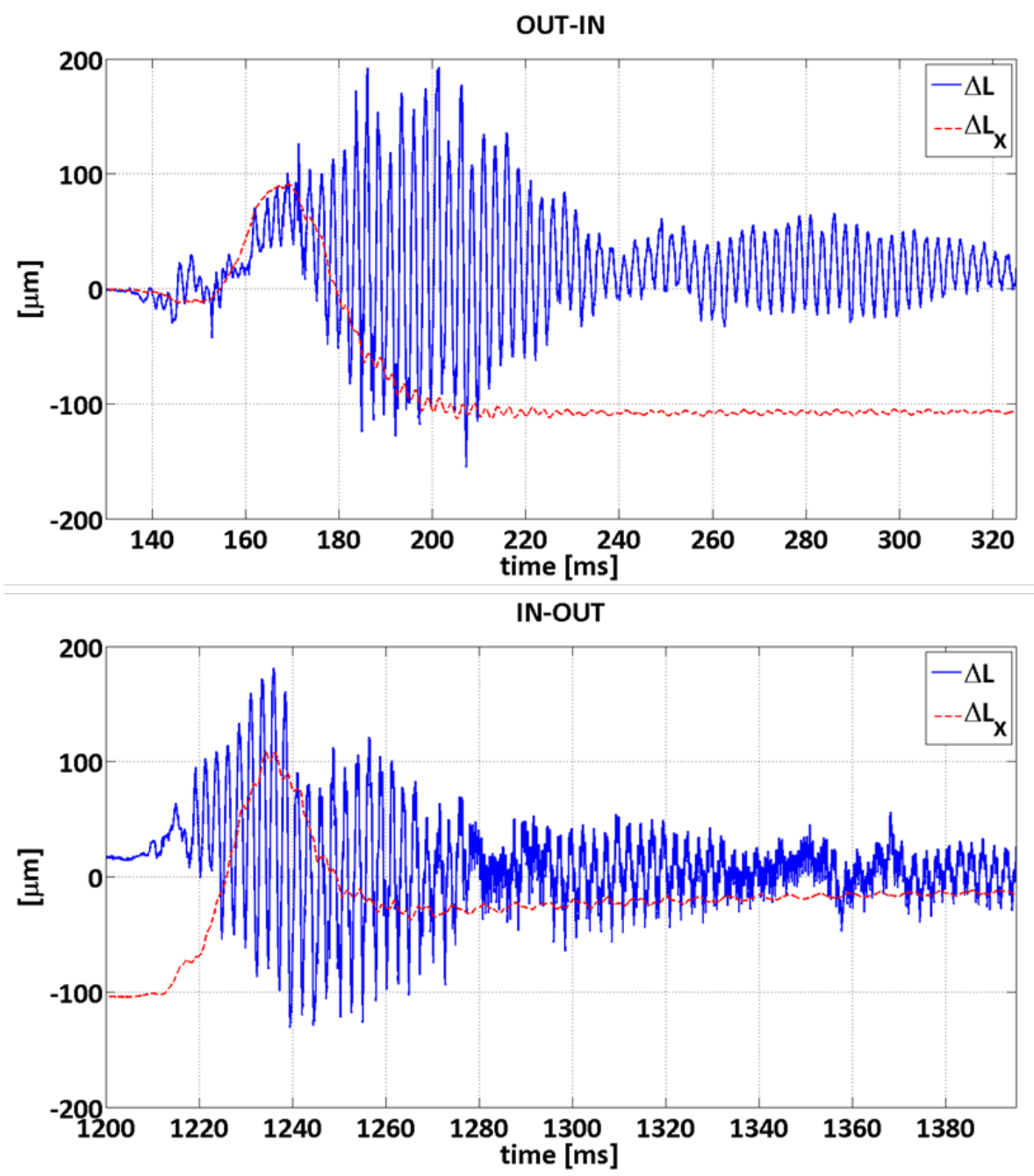

Fig. 21: Comparison between the wire elongation $\Delta \mathrm{L}$ and the fork tips separation $\Delta \mathrm{L}_{\mathrm{x}}$ during the OUT-IN phase (top) and the IN-OUT (bottom) phases. 
The tips separation $\Delta \mathrm{L}_{\mathrm{x}}$ has a direct consequence on the initial wire tension. Thus, the wire is actually a mechanical system with a variable parameter, which may yield parametric oscillations. Their amplitude might reach high values if a parametric resonance appeared. On the other hand, the accelerated rotation of the fork generates inertia forces which may also contribute to wire oscillations in the transverse dimension.

Thus, the real behaviour of the wire can be viewed as a result of a driven oscillation (associated with the inertia forces) and a parametric oscillation (associated with the wire tension changes).

A simple lumped-parameter model for the wire in order to illustrate this (driven+parametric) vibration is presented in the Appendix. Some more comments on the wire dynamics can be found in Section 5.

\section{Z-deflections}

The measured Z-deflections at the locations $Q_{4}^{R}$ and $Q_{4}^{L}$ were zero for all load values applied at the location $Q_{R}$ and $Q_{L}$. Therefore these loads do not produce any bending on the shaft along the $Y$ axis which could distort the measurements of the static deflections performed on the location $Q_{2}^{R, L}$ and $Q_{3}^{R, L}$.

Fig. 22 shows the Z-deflections at the location $Q_{1}^{R}$ and $Q_{1}^{L}$ obtained from the tension variations of the transversally sensitive strain gauges (GR2, GR3) and $(G L 2, G L 3)$, respectively using the corresponding calibration coefficients for the two phases of the scan cycle. If all gauges were correctly calibrated, $z\left(Q_{1}^{R}\right)$ should have the same value whether gauge GR2 or gauge GR3 is used. This should also be the case for $z\left(Q_{1}^{L}\right)$. The maximum difference between GR2 and GR3 or GL2 and GL3 is $50 \mu \mathrm{m}$. Remarkable is the agreement of the deflection given by the G3R and G3L of below $5 \mu \mathrm{m}$ for both scan directions OUT-IN and IN-OUT. This low difference of the G3 gauge results could be interpreted that their relative calibration accuracy is superior compared to the $\mathrm{G} 2$ gauges by a factor 10 . 

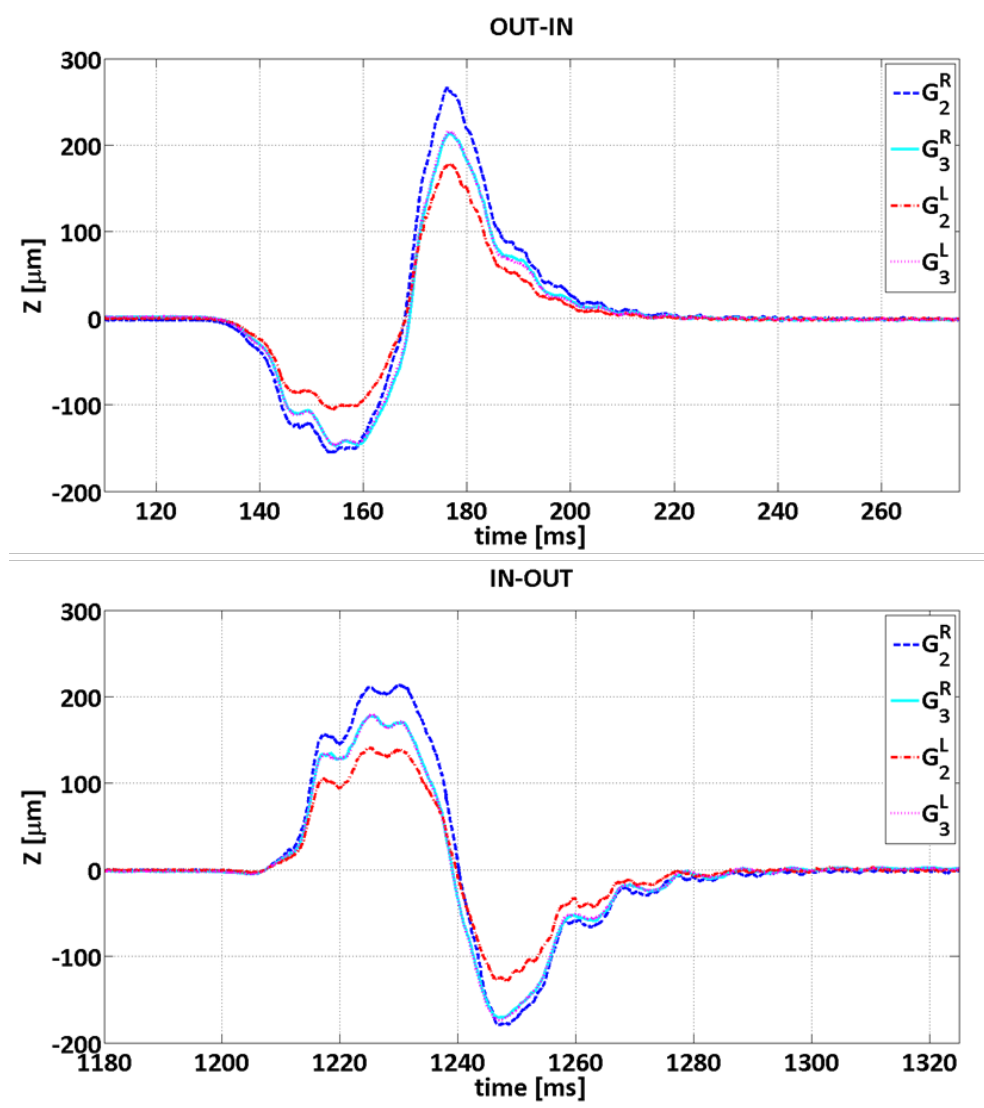

Fig. 22: Z-deflection at locations $Q_{1}^{R, L}$ calculated from the tension variations in the transversally sensitive strain gauges $(G 2, G 3)$ during the OUT -IN phase (top) and the IN -OUT (bottom) phases.

A close inspection of the measurements of Fig. 20 yield the following conclusions:

- $\quad z\left(Q_{1}^{R}\right)$ and $z\left(Q_{1}^{L}\right)$ obtained from gauges $G R 3$ and $G L 3$, respectively, are very close both in shape and amplitude. This consistency of the two independent measurements indicates the relative accuracy of the measurement system.

- $\quad z\left(Q_{1}^{R}\right)$ and $z\left(Q_{1}^{L}\right)$ obtained from gauges $G R 2$ and $G L 2$, respectively, show a similar qualitative behaviour but the signal amplitude is higher in the case of GR2, probably due to a higher sensitivity of GR2 as compared to that of GL2.

- $\quad z\left(Q_{2}^{L}\right)$ obtained from GL2 shows the same increasing and decreasing tendency as all the other curves but its amplitude is not as close to that of $z\left(Q_{2}^{L}\right)$ obtained from $G R 2$ as it should be. This probably indicates a defective bond between GL2 and the fork arm.

Fig. 23 shows the Z-deflections at points $Q_{1}^{R}$ and $Q_{1}^{L}$, obtained from the GR1 and GL1 gauges respectively, as a function of the shaft angular position along the two phases of the scan cycle (Fig.2). Both the right and the left fork arms show extremely close deflections, which is a proof of the consistency of the calibration coefficients. Typically the wire crosses the beam in the region of 110 degrees. 


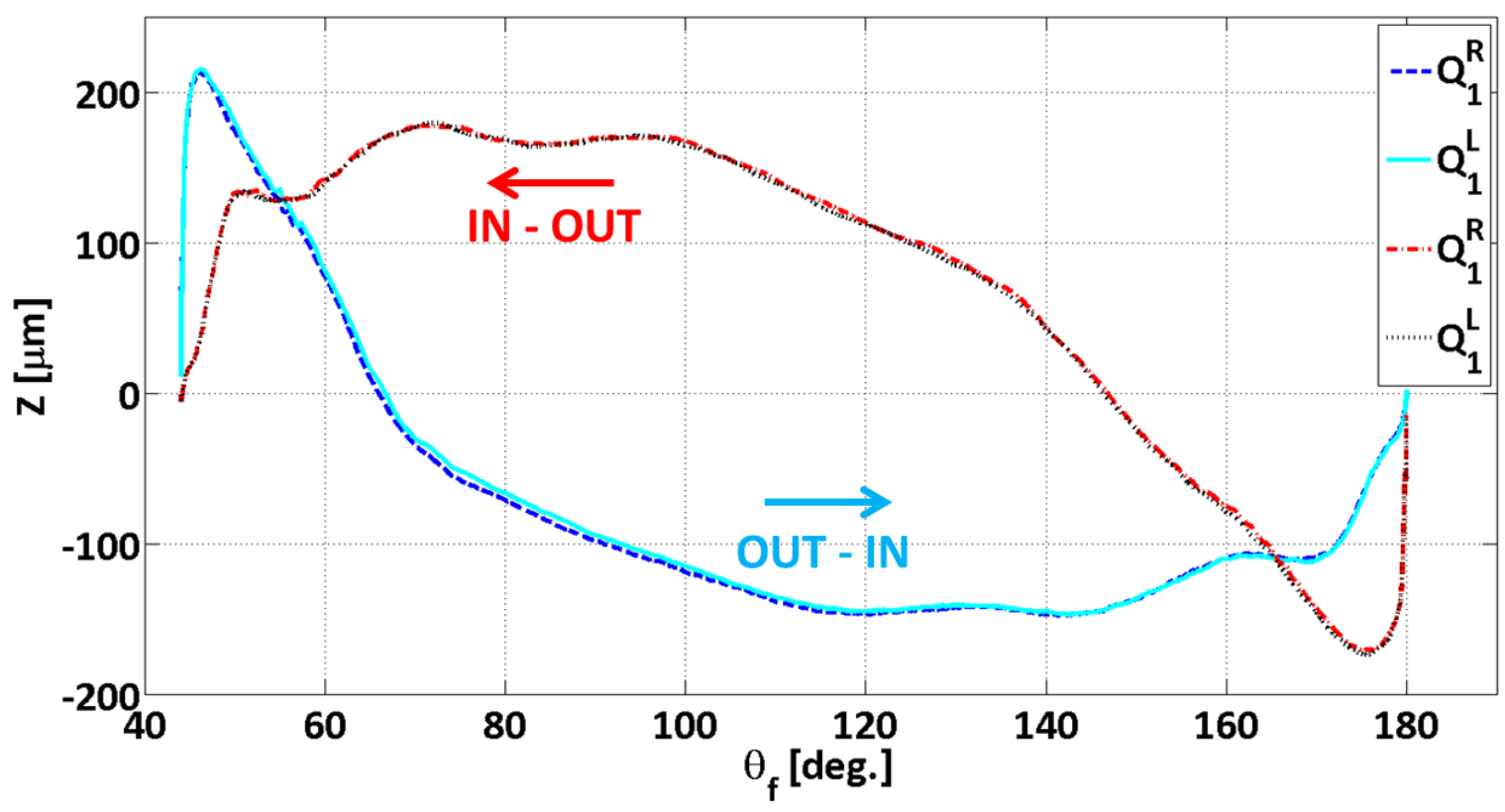

Fig. 23: Z-deflection of the fork tips (derived from the gauge measurements) for the two phases of the scan cycle as a function of the shaft angular coordinate.

The different behaviour on Z-deflection observed in Fig. 23 around the beam crossing (110 deg.) between OUT-IN and IN-OUT motions (IN-OUT motion shows the end of a plateau while OUTIN shows a steeper variation), can be understood through inspection of Fig. 17: for a same angular position of $110 \mathrm{deg}$. (located at $\mathrm{t}=160 \mathrm{~ms}$ and $\mathrm{t}=1236 \mathrm{~ms}$ for the OUT-IN and IN-OUT motions respectively), the values and trend of the angular acceleration are different, and that results in different Z-deflections.

\section{Error quantification}

The objective of this work was not to identify the error on the profile and position measurement for a given wire scanner. But to study the wire vibrations based on the piezoresistive effect of the wire itself. However in the following we try to quantify the maximum error in the profile and position measurements that this particular test scanner could provide.

From observation of Fig. 23 it is possible to make an assessment of the tip Z-deflection: around $-130 \mu \mathrm{m}$ for the OUT-IN motion and $150 \mu \mathrm{m}$ for the IN-OUT motions.

The assessment on the wire transversal vibrations requires further assumptions and analysis. Namely that the transversal shape of the wire describes a symmetry catenary between two $L_{x}$ distant points (see Fig. 24). 


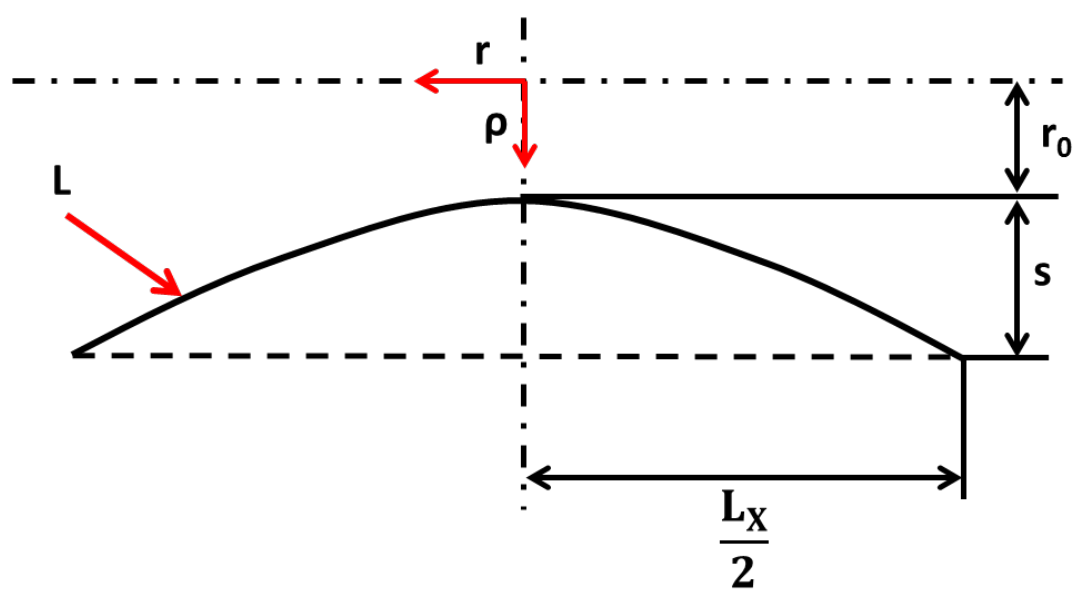

Fig. 24: Schematics used to define the catenary equation.

The equation of the catenary shown in Fig. 24 can be written as follows:

$$
\rho(r)=r_{0} * \cosh \left(\frac{r}{r_{0}}\right)
$$

where $r_{0}$ is a parameter which describes the curvature of the function.

The elongated wire length $L$, can be calculated as follows:

$$
L=\int_{-L_{x} / 2}^{L_{x} / 2} \sqrt{1+\left(\frac{d \rho}{d r}\right)^{2}} d r=\int_{-L_{x} / 2}^{L_{x} / 2} \sqrt{1+\sinh \left(\frac{r}{r_{0}}\right)^{2}} d r
$$

The value of the parameter $r_{0}$ can be determined from that equation. The value of the maximum wire sag s can be calculated as follows:

$$
s=\rho\left(\frac{L_{x}}{2}\right)-\rho(0)=r_{0} \cosh \left(\frac{L_{x}}{2 r_{0}}\right)-1
$$

Fig. 19 shows that the wire oscillations elongation amplitude $\Delta \mathrm{L}$, in the region of the beam crossing $(\mathrm{t}=160 \mathrm{~ms})$ for the OUT-IN motion is in the range of $50 \mu \mathrm{m}$, while the amplitude of the $\Delta \mathrm{Lx}$ oscillations is nearly 0 . Things are similar for the IN-OUT motion: the amplitude $\Delta \mathrm{L}(\mathrm{t}=1236 \mathrm{~ms})$ is in the range of $250 \mu \mathrm{m}$ and the $\Delta \mathrm{Lx}$ oscillation amplitude is almost 0 .

Table 6 summarizes the parameters and results used in the wire deflection assessment. 
Table 6: wire transversal deflection results.

\begin{tabular}{|c|c|c|c|c|}
\hline & & OUT-IN & IN-OUT & \\
\hline $\mathrm{L}_{0}$ & Wire initial length & 115 & 115 & {$[\mathrm{~mm}]$} \\
\hline$\Delta \mathrm{L}$ & Wire elongation & 0.050 & 0.250 & {$[\mathrm{~mm}]$} \\
\hline$\Delta \mathrm{L}_{\mathrm{x}}$ & Tips separation & $\sim 0$ & $\sim 0$ & {$[\mathrm{~mm}]$} \\
\hline $\mathrm{L}$ & Wire length & 115.050 & 115.250 & {$[\mathrm{~mm}]$} \\
\hline$L_{x}$ & Tips distance & 115 & 115 & {$[\mathrm{~mm}]$} \\
\hline$r_{0}$ & Catenary parameter & 1.12 & 0.5088 & -- \\
\hline S & Calculated wire sag & 1.476 & 3.253 & {$[\mathrm{~mm}]$} \\
\hline
\end{tabular}

Let's define $\mathrm{E}_{\mathrm{z}}^{\mathrm{F}}$ as the Z-deflection of the fork tip and $\mathrm{E}_{0}^{\mathrm{W}}$ as the maximum sag of the wire (which correspond to the previous value named as $s$ ). Therefore the maximum amplitude of a complete wire oscillation is $2 \mathrm{E}_{0}^{\mathrm{W}}$. In order to determine the beam size and position, the values provided by the angular sensor have to be projected in a plane perpendicular to the beam, i.e. "the projection plane". From the performed wire elongation measurements it is not possible to determine on which plane the wire is oscillating. However the most unfavourable configuration is when the wire transversal oscillation plane is parallel to the projection plane see Fig. 25.

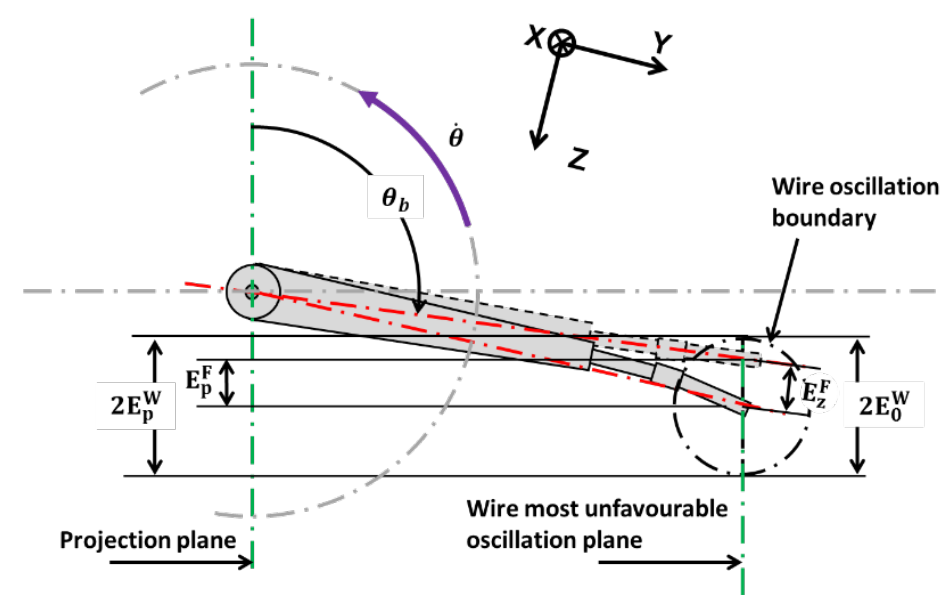

Fig. 25: Schematics of the wire position error projection. 
Due to the small size of the beam, $\mathrm{E}_{\mathrm{z}}^{\mathrm{F}}$ can be considered as constant along the whole beam crossing time. However the error due to the wire oscillation is not constant along this time. Let's assume that the time variation of the wire transversal deflection $\mathrm{E}^{\mathrm{W}}(\mathrm{t})$ obeys to a sinusoidal function of the form:

$$
\mathrm{E}^{\mathrm{W}}(\mathrm{t})=2 \mathrm{E}_{0}^{\mathrm{W}} \sin \left(\frac{2 \pi \omega}{2} \mathrm{t}\right)=2 \mathrm{E}_{0}^{\mathrm{W}} \sin (\pi \omega \mathrm{t})
$$

where $\omega$ stands for the wire oscillation frequency in $\mathrm{Hz}$. The projection of the fork tip deflection $\mathrm{E}_{\mathrm{p}}^{\mathrm{F}}$ can be calculated as:

$$
\mathrm{E}_{\mathrm{p}}^{\mathrm{F}}=\mathrm{E}_{\mathrm{z}}^{\mathrm{F}} \cos \left(\frac{\pi}{2}-\theta\right)
$$

where $\theta$ is the angular measured position. Therefore the total projected error $E_{p}(t)$ due fork tip and wire deflections can be calculated as follow:

$$
\mathrm{E}_{\mathrm{p}}(\mathrm{t})=\mathrm{E}_{\mathrm{p}}^{\mathrm{F}}+\mathrm{E}^{\mathrm{W}}(\mathrm{t})=\mathrm{E}_{\mathrm{z}}^{\mathrm{F}} \cos \left(\frac{\pi}{2}-\theta\right)+2 \mathrm{E}_{0}^{\mathrm{W}} \sin (\pi \omega \mathrm{t})
$$

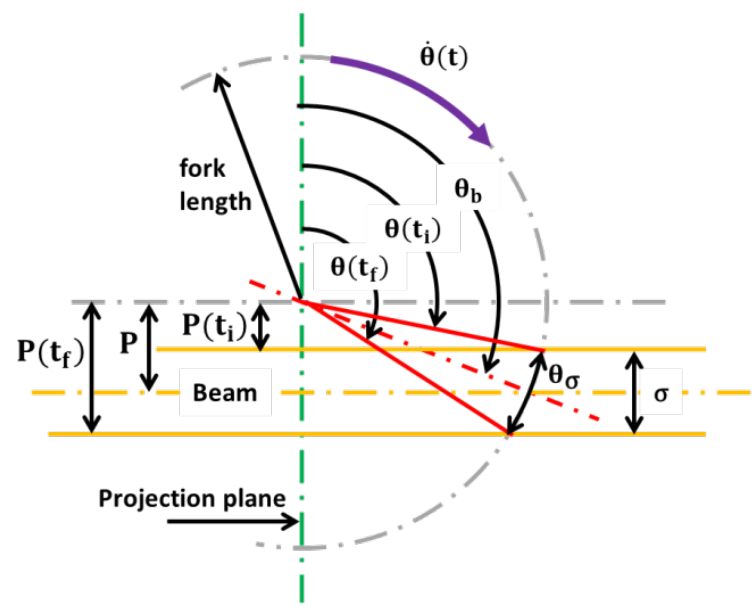

Fig. 26: Schematics of the beam profile and position measurements.

Based in Fig. 26, $t_{i}$ and $t_{f}$ can be defined as the initial and final crossing times of the wire with respect to a beam of size $\sigma$. Let's define $t_{\sigma}$ as the time that a wire traveling at a certain angular speed $\dot{\theta}(\mathrm{t})$ needs to traverse this beam, therefore: 


$$
\mathrm{t}_{\sigma}=\frac{\theta\left(\mathrm{t}_{\mathrm{f}}\right)-\theta\left(\mathrm{t}_{\mathrm{i}}\right)}{\dot{\theta}(\mathrm{t})}
$$

\section{Beam position measurement}

The measured position of the beam $\mathrm{P}_{\mathrm{m}}$ is defined as:

$$
P_{m}=\frac{P\left(t_{i}\right)+E_{p}\left(t_{i}\right)+P\left(t_{f}\right)+E_{p}\left(t_{f}\right)}{2}
$$

which can be written as:

$$
P_{m}=P+\frac{E_{p}\left(t_{i}\right)+E_{p}\left(t_{f}\right)}{2}
$$

where $\mathrm{P}$ is the real position of the beam. Therefore the beam position error $E_{p}^{P}$ is:

$$
E_{p}^{P}=\frac{E_{p}\left(t_{i}\right)+E_{p}\left(t_{f}\right)}{2}
$$

introducing Eq. (4.6) into Eq. (4.10) results in:

$$
\mathrm{E}_{\mathrm{p}}^{\mathrm{P}}=\frac{2 \mathrm{E}_{\mathrm{p}}^{\mathrm{F}}+2 \mathrm{E}_{0}^{\mathrm{W}}\left(\sin \left(\pi \omega \mathrm{t}_{\mathrm{i}}\right)+\sin \left(\pi \omega \mathrm{t}_{\mathrm{f}}\right)\right)}{2}
$$

From equation 4.11 and Fig. 27, it can be shown that the maximum value of $E_{p}^{P}$ does occur for a time interval $\left(t_{f}-t_{i}\right)$, such that $\sin \left(\pi \omega \frac{\left(t_{f}-t_{i}\right)}{2}\right)=1$, i.e, $\pi \omega \frac{\left(t_{f}-t_{i}\right)}{2}=\frac{\pi}{2}$. Therefore the maximum position error $\mathrm{E}_{\mathrm{p}_{\_} \text {max }}^{\mathrm{P}}$, can be calculated as follow:

$$
\mathrm{E}_{\mathrm{p}_{-} \max }^{\mathrm{P}}=\mathrm{E}_{\mathrm{p}}^{\mathrm{F}}+\mathrm{E}_{0}^{\mathrm{W}} \sin \left(\pi \omega\left(\frac{\mathrm{t}_{\sigma}}{2}\right)+\frac{\pi}{2}\right)
$$

\section{Width measurement}

The measured beam width, $\sigma_{m}$ can be calculated as the difference of the wire position projections at $t_{f}$ and $t_{i}$ instants, $P\left(t_{f}\right)$ and $P\left(t_{i}\right)$ respectively.

$$
\sigma_{m}=P\left(t_{f}\right)+E_{p}\left(t_{f}\right)-P\left(t_{i}\right)-E_{p}\left(t_{i}\right)
$$




$$
\sigma_{m}=\sigma+E_{p}\left(t_{f}\right)-E_{p}\left(t_{i}\right)
$$

Therefore the beam size error $\mathrm{E}_{\mathrm{p}}^{\sigma}$ is:

$$
E_{p}^{\sigma}=E_{p}\left(t_{f}\right)-E_{p}\left(t_{i}\right)
$$

introducing Eq. (4.6) into Eq. (4.15) and considering that $\mathrm{E}_{\mathrm{p}}^{\mathrm{F}}$ can be treated as constant along the beam crossing, results in:

$$
\mathrm{E}_{p}^{\sigma}=2 \mathrm{E}_{0}^{\mathrm{W}}\left(\sin \left(\pi \omega t_{f}\right)-\sin \left(\pi \omega t_{i}\right)\right)
$$

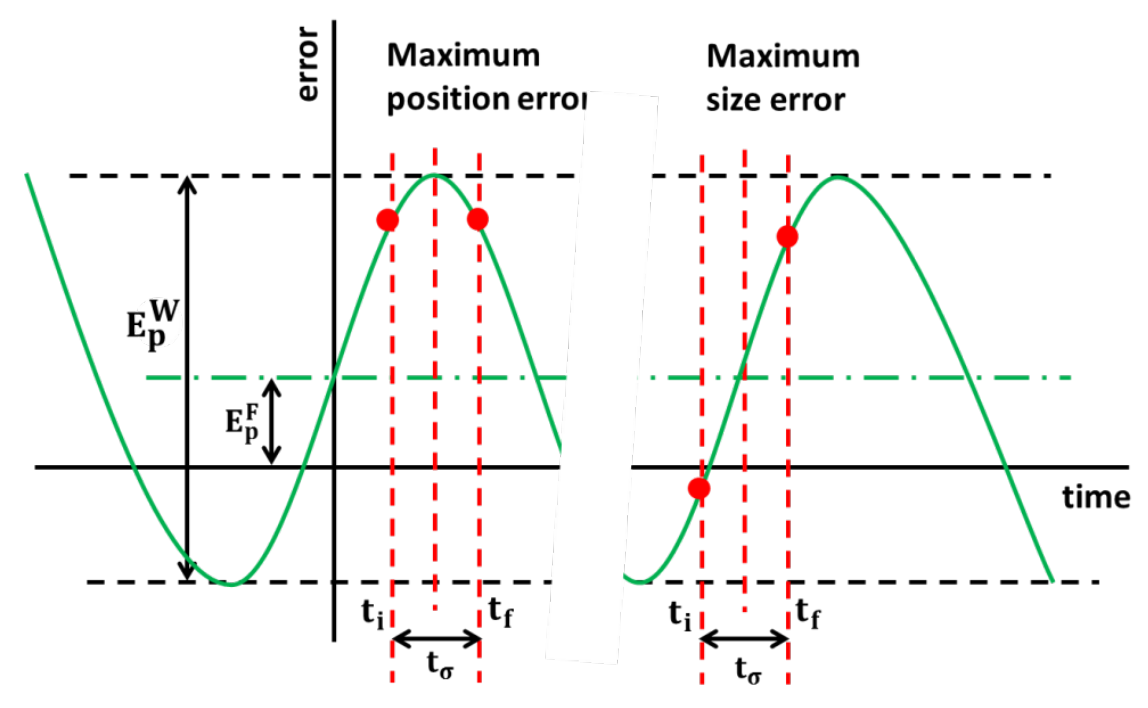

Fig. 27: Time interval schematic for the maximum position and size error locations.

Again from Fig. 27 and equation 4.16, the maximum value of $E_{p}^{\sigma}$ does occur for a time interval $\left(t_{f}-t_{i}\right)$, such that $\sin \left(\pi \omega \frac{\left(t_{f}-t_{i}\right)}{2}\right)=0$, i.e, $\pi \omega \frac{\left(t_{f}-t_{i}\right)}{2}=(0, \pi)$. In this case, the maximum error for the beam size measurement $\mathrm{E}_{\mathrm{p} \_ \text {max }}^{\sigma}$ is:

$$
\mathrm{E}_{\mathrm{p}}^{\sigma}=4 \mathrm{E}_{0}^{\mathrm{W}} \sin \left(\pi \omega\left(\frac{\mathrm{t}_{\sigma}}{2}\right)\right)
$$

Table 7 shows the beam position and beam width maximum errors calculated for different beam sizes. For this calculation the value of the angular speed at the beam crossing time interval has been considered as constant, its value is extracted from Fig. 17. 
Table 7: maximum calculated error in size and position

\begin{tabular}{|c|c|c|c|c|c|c|c|c|}
\hline & & \multicolumn{3}{|c|}{ OUT - IN } & \multicolumn{3}{c|}{ IN - OUT } & \\
\hline$\sigma$ & Beam size & 2 & 1 & 0.2 & 2 & 1 & 0.2 & {$[\mathrm{~mm}]$} \\
\hline$\dot{\theta}$ & Beam cross. speed & 100 & 100 & 100 & 110 & 110 & 110 & {$[\mathrm{rad} / \mathrm{s}]$} \\
\hline$\theta_{\mathrm{b}}$ & Beam cross. ang. & 110 & 110 & 110 & 110 & 110 & 110 & {$[\mathrm{deg}]$} \\
\hline $\mathrm{t}_{\sigma}$ & Beam cross. interval & 0.129 & 0.065 & 0.013 & 0.118 & 0.059 & 0.018 & {$[\mathrm{~ms}]$} \\
\hline $\mathrm{E}_{\mathrm{p}_{\_} \text {max }}^{\mathrm{P}}$ & Max. pos. error & -1.60 & -1.60 & -1.61 & 3.39 & 3.40 & 3.40 & {$[\mathrm{~mm}]$} \\
\hline$\delta \mathrm{E}_{\mathbf{p}_{\_} \text {max }}^{\mathrm{P}}$ & Relative max. pos. error & 2.85 & 2.86 & 2.86 & -6.05 & -6.06 & -6.07 & {$[\%]$} \\
\hline $\mathrm{E}_{\mathrm{p}_{\_} \text {max }}^{\sigma}$ & Max. size error & 0.48 & 0.24 & 0.05 & 0.96 & 0.48 & 0.10 & {$[\mathrm{~mm}]$} \\
\hline$\delta \mathrm{E}_{\mathrm{p}_{\_} \text {max }}^{\sigma}$ & Relative max. size error & 24.04 & 24.06 & 24.07 & 48.18 & 48.22 & 48.23 & {$[\%]$} \\
\hline
\end{tabular}

The error values shown in Table 7 are very high, especially in regards to the measure of width, but it has to be taken into account that this measurements has been performed in a test scanner with more than 250000 scan cycles (typically operating scanner do not perform more than 50000 cycles before to be replaced).

\section{COMMENTS ON THE WIRE DYNAMICS}

As mentioned in the previous section, the wire oscillation and deflection are driven by the inertia forces associated with shaft rotation, and the likely parametric oscillations are associated with the wire tension changes caused by the fork tips deflections in the $\mathrm{X}$ direction.

In order to highlight the causes of the wire oscillations, a modal analysis of the (fork+wire) system has been performed through the FE model. The two first natural vibration modes associated with the fork X-deflection are shown in Fig. 28. The first X-mode has a frequency of $151 \mathrm{~Hz}$ and consists on the symmetrical X-deflection of the fork tips (SX-mode). In that case, the wire keeps its length constant.

The second X-mode is located at $382 \mathrm{~Hz}$ and consists on an antisymmetrical X-deflection of the tips (AX-mode). This mode may lead to instability of the wire straight shape thus generating transverse parametrically-driven oscillations on the wire. 

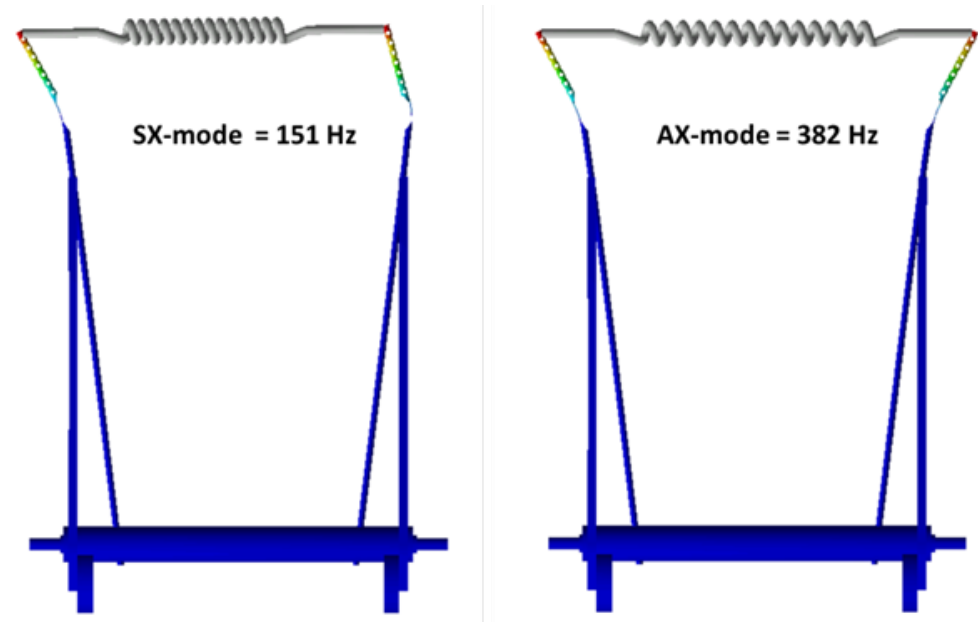

Fig. 28: Symmetric (left) and Antisymmetric (right) X-modes of vibration obtained through the modal analysis performed with the FE model.

Fig. 29 shows the FFT performed on the X-deflections at the location $Q_{1}^{R}$ and $Q_{1}^{L}$ (plotted in Fig. 18.) Two peaks appear at frequencies $154 \mathrm{~Hz}$ and $404 \mathrm{~Hz}$. These two frequencies match the two frequencies of $151 \mathrm{~Hz}$ and $382 \mathrm{~Hz}$ shown by the modal analysis performed on the FE model with a relative error of $1.9 \%$ and $5.7 \%$ respectively. The differences between model and measurements are related to the fact that the mass of the wire soldering in the model is heavier as on the real fork.

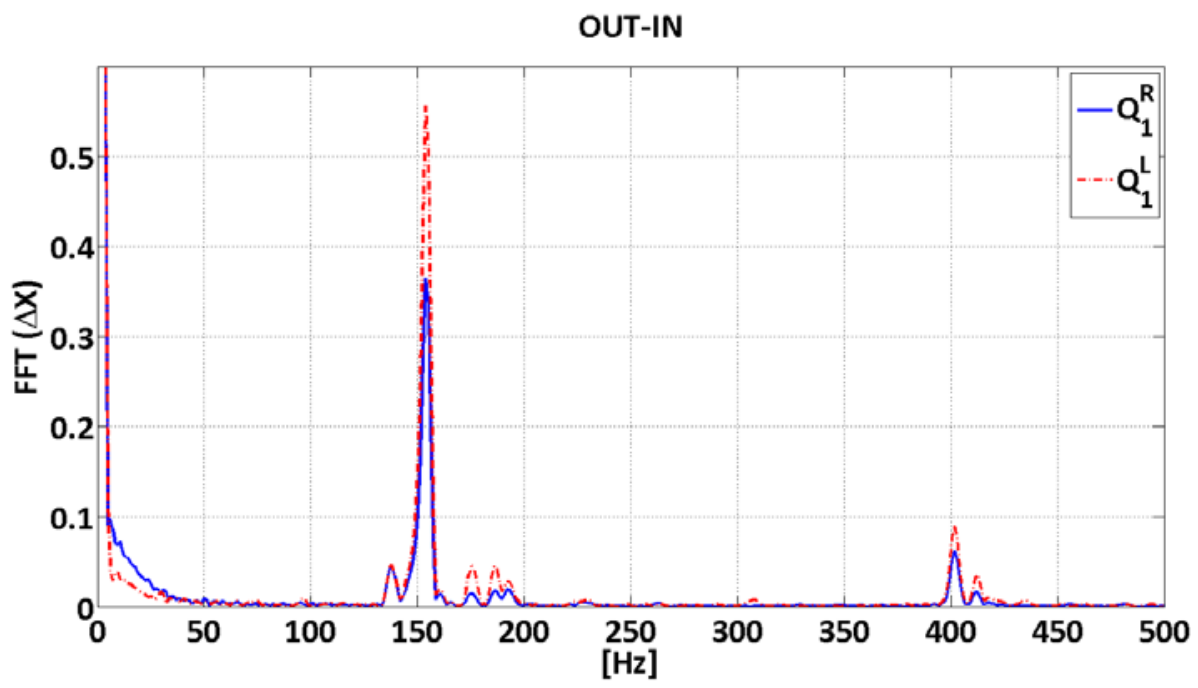

Fig. 29: Spectra of the X-deflections of the fork tips shown in Fig. 18.

Fig. 30 shows the FFT performed on the wire elongation signal plotted in Fig. 19. The highamplitude peak appears around $400 \mathrm{~Hz}$ confirms the hypothesis of a parametric vibration of the wire generated by the AS-mode. 


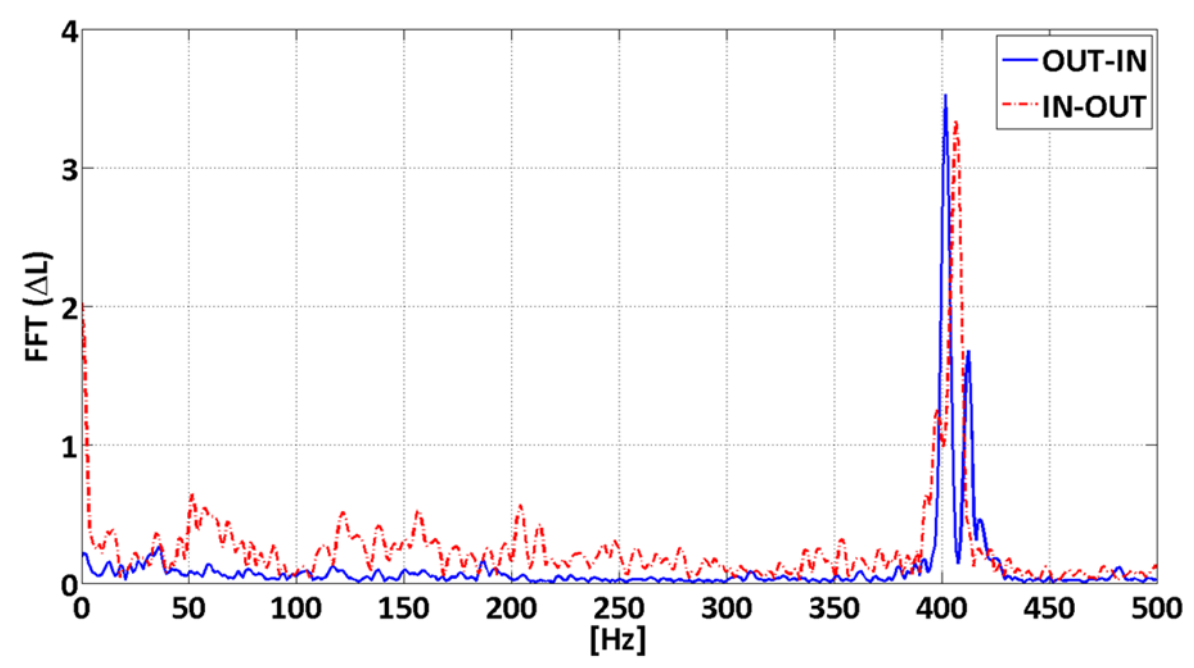

Fig. 30: Spectrum of the wire elongation $\Delta \mathrm{L}$ plotted in Fig. 19.

\section{CONCLUSIONS}

A vibration measurement system has been developed and tested. The results of the measurements show that this system allows the visualization of the vibrational behaviour of the existing wire scanner. It has been proved that the measurements based on the wire piezoresistive effect provide consistent information and allows the determination of the vibrational behaviour of such a system. Additionally it has been demonstrated that it is possible to quantify the maximum error induced by these vibrations in the beam profile and position measurement.

A hybrid calibration procedure using experimental measurement and FE has been developed in order to overcome the difficulty of the calibration under a dynamic and non-linear acceleration field.

The vibration measurement system based in the wire piezoresistive effect can be easily implemented in existing wire scanner devices as only an external signal measurement system is required.

The further work will be focused on the implementation of the vibrational measurement system in the new wire scanner design. More accurate theoretical models will also be developed to verify the hypothesis of the role of parametric excitation in the wire oscillations. 


\section{Acknowledgements}

I would like to thank all my colleagues of the $\mathrm{BI} / \mathrm{BL}$ section for their constant support and help concerning the electronic issues, especially Carlos Pereira and Jose Sirvent, who developed the electronic readout system under the supervision of Jonathan Emery.

\section{APPENDIX}

The simplest model for the wire is a lumped-parameter (or discrete) model (DM). It consists on a 2 Degrees-of-Freedom (DoF) where the wire is represented as two linear (spring+damper) sets (with constants $k$ and $c$ respectively) connected through a mass point $P$ (Fig. A1).

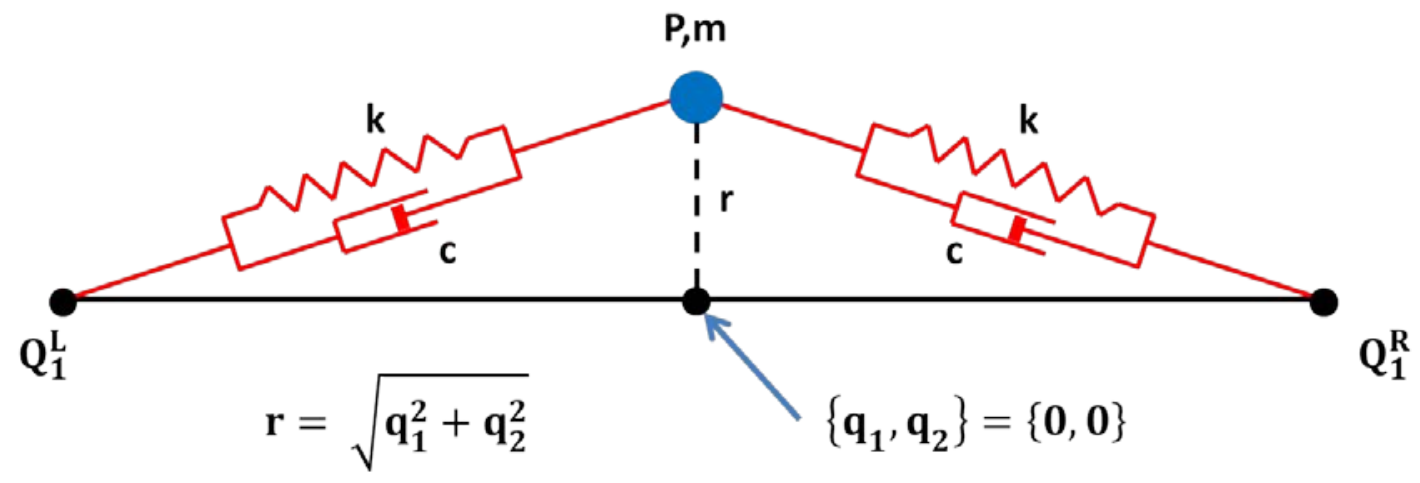

Fig. A.1: Lumped-parameter model for the vibrating wire.

If the fork were rigid, Newton's second law applied to the DM in a reference frame translating with the fork tips (Translating Frame TF) would have to include drag inertia forces (Fig. A2).

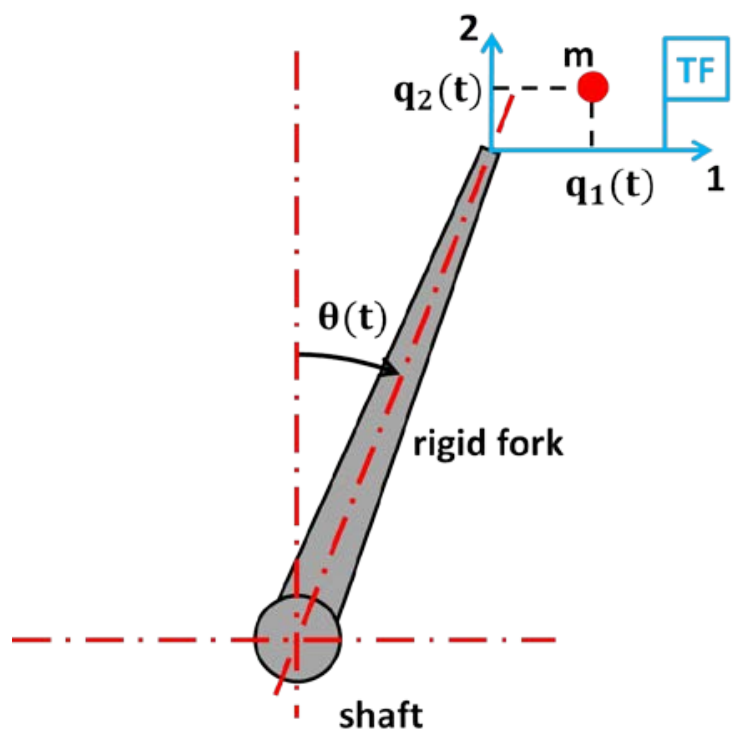

Fig. A.2: Definition of the Translating Frame (TF) where the wire dynamics are studied. 
In the $T F$, the motion of the mass point is described through coordinates $\left(q_{1}, q_{2}\right)$. In the reference rest configuration, $\left(q_{1}, q_{2}\right)=(0,0)$. The fork motion in the Ground Frame (GF) is described through just one angular coordinate $\theta$. In the reference rest configuration, $\theta=0$.

Newton's second law applied to the particle $P$ in that frame states:

$$
\bar{F}_{\mathrm{R}}+\overline{\mathrm{F}}_{\mathrm{L}}+\overline{\mathrm{F}}_{\mathrm{drag}}=\mathrm{m} \overline{\mathrm{a}}_{\mathrm{TF}}(\mathrm{P}) \text {, }
$$

where $\bar{F}_{d r a g}$ is the drag inertia force, and $\bar{F}_{R, L}$ are the forces associated with the (spring+damper) sets at both sides of the mass point. As the problem is symmetrical: $F_{R}=F_{L}=F$. The weight is negligible as compared to all the other forces acting on the mass point P. Eq.(A.1) becomes:

$$
m\left\{\begin{array}{c}
\ddot{q}_{1} \\
\ddot{q}_{2}
\end{array}\right\}+\frac{2 F}{\rho}\left\{\begin{array}{c}
q_{1} \\
q_{2}
\end{array}\right\}=m H\left\{\begin{array}{c}
\dot{\theta}^{2} \sin \theta-\ddot{\theta} \cos \theta \\
\dot{\theta}^{2} \cos \theta+\ddot{\theta} \sin \theta
\end{array}\right\} \text {, with } F=T_{0}+k\left(\rho-\frac{L_{0}}{2}\right)+c \dot{\rho}
$$

where $2 \rho=2 \sqrt{q_{1}^{2}+q_{2}^{2}+\left(L_{0} / 2\right)^{2}}$ is the total wire length and $T_{0}$ is the spring tension at the rest configuration.

If the transverse wire deformations are small (compared to the wire length), Eq.(A.2) simplifies to:

$$
m\left\{\begin{array}{c}
\ddot{q}_{1} \\
\ddot{q}_{2}
\end{array}\right\}+\frac{4 T_{0}}{L_{0}}\left\{\begin{array}{l}
\mathrm{q}_{1} \\
\mathrm{q}_{2}
\end{array}\right\}=m H\left\{\begin{array}{l}
\dot{\theta}^{2} \sin \theta-\ddot{\theta} \cos \theta \\
\dot{\theta}^{2} \cos \theta+\ddot{\theta} \sin \theta
\end{array}\right\}
$$

The left hand side (Ihs) corresponds to a harmonic oscillator with a natural angular frequency

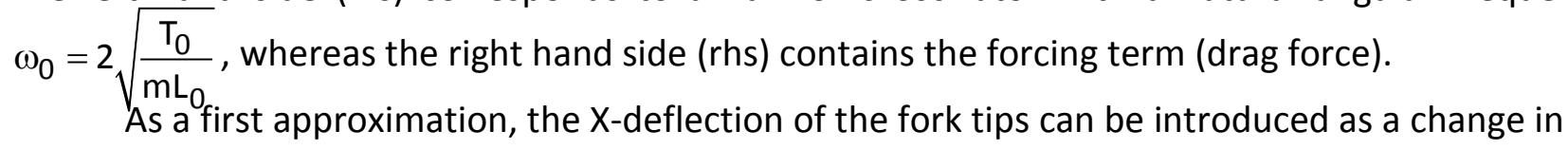
the spring tension:

$$
\mathrm{m}\left\{\begin{array}{l}
\ddot{\mathrm{q}}_{1} \\
\ddot{\mathrm{q}}_{2}
\end{array}\right\}+\frac{4 \mathrm{~T}(\mathrm{t})}{\mathrm{L}_{0}}\left\{\begin{array}{c}
\mathrm{q}_{1} \\
\mathrm{q}_{2}
\end{array}\right\}=\mathrm{mH}\left\{\begin{array}{c}
\dot{\theta}^{2} \sin \theta-\ddot{\theta} \cos \theta \\
\dot{\theta}^{2} \cos \theta+\ddot{\theta} \sin \theta
\end{array}\right\} \text {, with } \mathrm{T}(\mathrm{t})=\mathrm{T}_{0}+\mathrm{k} \Delta \mathrm{L}_{\mathrm{x}}(\mathrm{t})
$$

Each component in Eq.(A.4) corresponds to a 1 DoF oscillator undergoing a driven excitation and a parametric excitation:

$$
\ddot{q}_{i}+\omega^{2}(t) q_{i}=f_{i}(t) .
$$




\section{REFERENCES}

Ch. Steinbach, M. van Rooij, A scanning wire beam profile monitor, IEEE Transactions on Nuclear Science, NS-32 (1985), 1920-1922

[2]

J. Bosser, Beam Instrumentation, CERN, (1996) $253-255$.

[3]

F. Roncarolo, Accuracy of the Transverse Emittance Measurements of the CERN Large Hadron Collider, Doctoral Thesis, Ecole Polytechnique Fédérale de Lausanne, 2005.

[4] J. Koopman, A.G Ollacarizqueta, A. Lokhovitskiy, Fast wire scanner calibration, Proceedings of the DIPAC'09 Conference (2009), 170-172

[5] W. Blokland, G. Vogel, J. Dey, A new flying wire system for the Tevatron, Particle accelerator conference, (1997), 2032-2034

J. Bosser, J. Camas, L. Evans, G. Ferioli, R. Hopkins, J. Mann, O. Olsen, Transverse emittance measurement with a rapid wire scanner at the CERN SPS, Nuclear Instruments and Methods in Physics Research A 235(3) (1985) 475-480.

[7] R. Veness, N. Chritin, B. Dehning, J. Emery, J. Herranz Alvarez, M. Koujili, S. Samuelsson, J.L. Sirvent, Design of a high-precision fast wire scanner for the SPS at CERN Proceedings of IBIC2012, (2012)

[8] N. lida, T. Suwada, Y. Funakoshi, T. Kawamoto, M. Kikuchi, A method for measuring vibrations in wire scanner beam profile monitors, Proceedings of APAC'98, (1998), 546-548

[9] R. Shepherd, Strain measurement using vibrating-wire gages, Experimental Mechanics, 4 (8) (1964) 244-248

[10] S. Dan Mihai, Handbook of Force Transducers, Springer (2012) 203-205

[11] S.G. Arutunian, N.M. Dobrovolski, M.R. Mailian, I.G. Sinenko, I.E. Vasiniuk, Vibrating Wire Scanner for beam profile monitoring, Proceedings of the 1999 Particle Accelerator Conference, (1999), 2105-2107

[12] S. Arutunian, A. Avetisyan, N. Dobrovolski, M. Mailian, I. Vasiniuk, K. Wittenburg, R. Reetz, Problems of installation of vibrating wire scanners into accelerator vacuum chamber, Proceedings of EPAC, (2002) 1837-1839 


\section{FIGURE CAPTIONS}

Fig. 1: Schematics of the wire scanner instrument.

Fig. 2: Schematics of the PS scanner scan cycle from OUT to IN (left) and from IN to OUT (right) positions.

Fig. 3: Schematics of the wire deflection in the measurement chain.

Fig. 4: Experimental setup: actuator (1), fork (2), vacuum tank (3), Wheatstone bridge and amplifier for wire resistance variations (4), Wheatstone bridge and amplifier for strain gauges (5), oscilloscope (6), power supplies (7).

Fig. 5: Semiconductor strain gauge detail bonded in the PS scanner arm.

Fig. 6: Location of the semiconductor strain gauges on the PS fork $\left(G_{n}^{R, L}\right)$, location of points whose deflection has been measured $\left(Q_{n}^{R, L}\right)$, and definition of the coordinate system.

Fig. 7: Experimental setup used to measure wire resistance variation as a function of the wire elongation.

Fig. 8: Change of wire elongation as funtion of voltage change.

Fig. 9: Typical result of fork tip deflections $\Delta X\left(Q_{1}^{R}\right)$ and $\Delta X\left(Q_{1}^{L}\right)$ vs. tension variations on $G L 1$ and GR1, respectively.

Fig. 10: PS fork and applied load at $Q_{R, L}$ locations. $Q_{n}^{R, L}$ and $G_{n}^{R, L}$ are the measurement points and the gauge locations (as in Fig. 6).

Fig. 11: Finite Element model of the fork wire arrangement.

Fig. 12: Measured (exp) and calculated (FE) static deflections at points $Q_{2}^{R, L}$.

Fig. 13: Typical result of calculated fork tip deflection vs. calculated strain for values of angular acceleration in the range $(0-12000) \mathrm{rad} / \mathrm{s}^{2}$.

Fig. 14: Typical result of correlation between the tension recorded by gauges $G R 2$ and $G L 2$, and the strain at points $G_{3}^{R, L}$ calculated with the FE model.

Fig. 15: Flow diagram showing the calibration procedure. The diagram is subdivided in a static part (measurements and FE) and a dynamic part (only FE based). 
Fig. 16: Schematics of the fork deflections with resulting wire elongation and location of the strain gauges.

Fig. 17: Typical measured motion patterns for a full scan motion cycle: angular position (a), angular velocity (b) and angular acceleration (c).

Fig. 18: X-deflection of the fork tips (locations $Q_{1}^{R, L}$ ) for the OUT-IN (top) and the IN-OUT (bottom) phases, calculated from the tension variations at gauges GR1 and GL2.

Fig. 19: Values of the tip deflection for a gentle manual rotation.

Fig. 20: Schematics of the phenomenon causing the offset signals between OUT and IN.

Fig. 21: Comparison between the wire elongation $\Delta \mathrm{L}$ and the fork tips separation $\Delta \mathrm{L}_{\mathrm{x}}$ during the OUT-IN phase (top) and the IN-OUT (bottom) phases.

Fig. 22: Z-deflection at the location $Q_{1}^{R, L}$ calculated from the tension variations in the transversally sensitive strain gauges $(G 2, G 3)$ during the OUT -IN phase (top) and the IN-OUT phases (bottom).

Fig. 23: Z-deflection of the fork tips (derived from the gauge measurements) for the two phases of the scan cycle as a function of the shaft angular coordinate.

Fig. 24: Schematics used to define the catenary equation.

Fig. 25: Schematics of the wire position error projection.

Fig. 26: Schematics of the beam profile and position measurements.

Fig. 27: Time interval schematic for the maximum position and size error locations.

Fig. 28: Symmetric (left) and Antisymmetric (right) X-modes of vibration obtained through the modal analysis performed with the FE model.

Fig. 29: Spectra of the X-deflections of the fork tips shown in Fig. 18.

Fig. 30: Spectrum of the wire elongation $\Delta \mathrm{L}$ plotted in Fig. 19.

Fig. A.1: Lumped-parameter model for the vibrating wire.

Fig. A.2: Definition of the Translating Frame (TF) where the wire dynamics are studied. 